\title{
VECTOR DECOMPOSITIONS OF ROTATIONS
}

\section{DANAIL BREZOV, CLEMENTINA MLADENOVA AND IVAÏLO MLADENOV}

Presented by Ivaïlo M. Mladenov

Abstract. Here we derive analytic expressions for the scalar parameters which appear in the generalized Euler decomposition of the rotational matrices in $\mathbb{R}^{3}$. The axes of rotations in the decomposition are almost arbitrary and they need only to obey a simple condition to guarantee that the problem is well posed. A special attention is given to the case when the rotation is decomposable using only two rotations and for this case quite elegant expressions for the parameters were derived. In certain cases one encounters infinite parameters due to the rotations by an angle $\pi$ (the so called half turns). We utilize both geometric and algebraic methods to obtain those conditions that can be used to predict and deal with various configurations of that kind and then, applying l'Hôpital's rule, we easily obtain the solutions in terms of linear fractional functions. The results are summarized in two Tables and a flowchart presenting in full details the procedure.

\section{Contents}

1 Introduction $\quad 60$

2 The Generic Case $\quad 64$

3 The Symmetric Case $\quad 69$

4 Decomposition Into Two Rotations 71

5 Cases Involving a Half Turn $\quad \mathbf{7 5}$

5.1 The Case of Two Rotations . . . . . . . . . . . . . . . . . . 76

5.2 The Case of Three Rotations . . . . . . . . . . . . . . . . . . 79

6 The Identity Transformation $\quad 84$

$\begin{array}{llr}7 & \text { Examples } & 86\end{array}$

8 Concluding Remarks $\quad 91$

References $\quad 42$ 


\section{List of Symbols and Notations}

$$
\begin{aligned}
& \mathbf{x}, \tilde{\mathbf{x}}, \stackrel{\circ}{\mathbf{c}}_{1}, \stackrel{\circ}{\mathbf{c}}_{2}, \stackrel{\circ}{\mathbf{c}}_{3} \\
& \hat{\mathbf{c}}_{1}, \hat{\mathbf{c}}_{2}, \hat{\mathbf{c}}_{3}, \hat{\mathbf{n}} \\
& \mathbf{c}, \tilde{\mathbf{c}}, \mathbf{c}_{1}, \mathbf{c}_{2}, \mathbf{c}_{3} \\
& \mathbf{x} \otimes \mathbf{z}^{t} \\
& \times \\
& (\cdot, \cdot) \\
& \varepsilon_{i j k} \\
& \mathbf{c}^{\times} \\
& \mathcal{I} \\
& \mathcal{P}_{\alpha}, \mathcal{P}_{\alpha}^{\perp} \\
& \mathcal{R}(\mathbf{c})=\mathcal{R}, \mathcal{R}\left(\mathbf{c}_{k}\right)=\mathcal{R}_{k} \\
& \mathcal{O}(\hat{\mathbf{n}}), \mathcal{O}\left(\hat{\mathbf{c}}_{k}\right)=\mathcal{O}_{k} \\
& \lambda_{k} \\
& \mu \\
& u, v, w, \tau \\
& \psi_{1}, \psi_{2}, \psi_{3} \\
& \varphi, \phi, \theta_{k}, \beta_{k}, \gamma_{i j} \\
& \kappa_{i j}=\left(\hat{\mathbf{c}}_{i}, \hat{\mathbf{c}}_{j}\right) \\
& \sigma_{i j}=\left(\hat{\mathbf{c}}_{i}, \mathcal{R}(\mathbf{c}) \hat{\mathbf{c}}_{j}\right) \\
& \rho_{k}=\left(\hat{\mathbf{n}}, \hat{\mathbf{c}}_{k}\right), \quad \varepsilon_{i j k} \tilde{\rho}_{k}=\left(\hat{\mathbf{c}}_{i} \times \hat{\mathbf{c}}_{j}, \hat{\mathbf{n}}\right) \\
& \omega=\left(\hat{\mathbf{c}}_{1}, \hat{\mathbf{c}}_{2} \times \hat{\mathbf{c}}_{3}\right) \\
& \xi_{k}=\tau \rho_{k}
\end{aligned}
$$

\section{Introduction}

Decomposing an arbitrary rotational motion into two or three successive rotations about initially given axes is a problem, that has been present both in mathematics and the applications for centuries and yet it appears far from being trivial, especially in the case when the chosen axes are non-orthogonal [5].

The necessity of such decomposition is dictated by the practical needs of industry and engineering sciences. It is worth mentioning that the factorization of orthogonal matrices plays an important role in modern navigation and control of aircrafts, submarines, and communication satellites [21], crystallography and diffractometry [6], nuclear magnetic resonance [30], digital image processing [14] and optics [27]. In any of these areas it is necessary to perform several successive displacements in order to obtain the desired settings. 
There are many partial results in the literature, based on different techniques, but none of them seems to cover the subject extensively. Here we present a simple and yet powerful technique that resolves also the "pathological" cases, consistently avoided by other authors.

The starting point we chose for the present article is a theorem due to Euler, stating that each orthogonal transformation in odd dimensional real space has an invariant axis. Among its many proofs, one particularly simple resorts on the fact that the eigenvalues of orthogonal operators lie on the unit circle $\mathrm{U}(1)$. It is quite easy to verify this with the help of the exponential map and the well known result that the eigenvalues of skew-symmetric matrices that constitute the Lie algebra of the orthogonal group, are purely imaginary numbers. In other words, all zeroes of the polynomial

$$
\operatorname{det}(\mathcal{R}-\lambda \mathcal{I})=0
$$

are of modulus one.

Since $\mathcal{R}$ acts as automorphism of $\mathbb{R}^{2 m+1}$, the above polynomial is of degree $2 m+1$ and must have at least one real root since the complex ones come in pairs (all coefficients being real) and therefore it has to be either +1 or -1 . In the case of only one real root, that is the generic case in $\mathbb{R}^{3}$, we have pure rotation if the sign is plus, and the corresponding eigenvector determines the axis of that rotation. In the case $\lambda_{1}=-1$, we are dealing with a composition of rotation and reflection, and the eigenvector is interpreted as normal to the plane of reflection. More generally, both +1 and -1 may be multiple roots as in the case of symmetric orthogonal matrices where all eigenvalues are real. Then $\mathcal{R}$ represents a special orthogonal transformation, or pure rotation, only if the multiplicity $\mu$ of -1 is even, since $\operatorname{det} \mathcal{R}=(-1)^{\mu}$.

In this article we focus entirely on the three dimensional case, starting with $\mu=0$. Then $\mathcal{R}$ is either the identity operator if $\lambda_{1}=\lambda_{2}=\lambda_{3}=1$, or a generic rotation with $\lambda_{1}=1, \lambda_{2}=\bar{\lambda}_{3} \in \mathrm{U}(1)$, whose axis is given by the unit eigenvector $\hat{\mathbf{n}}$, corresponding to $\lambda_{1}$. The actual rotation by an angle $\varphi \neq k \pi, k \in \mathbb{Z}$ takes place in the plane $\alpha$, perpendicular to $\hat{\mathbf{n}}$.

The case $\mu=1$ corresponds to either pure reflection (if $\lambda_{2}=\lambda_{3}=1$ ) with respect to $\alpha$, or a composition of reflection and rotation by $\varphi \neq k \pi$ if $\lambda_{2}=\bar{\lambda}_{2} \in \mathrm{U}(1)$.

When $\mu=2\left(\lambda_{1}=1, \lambda_{2}=\lambda_{3}=-1\right)$ we deal with non-trivial symmetric orthogonal operators, also called "half turns" since they rotate by an angle $\pi$ in $\alpha$. Note that these operators turn to pure reflections when multiplied by -1 , so they share some common properties with reflections, which is certainly true to a lower extent for all orthogonal transformations since they are in fact compositions of reflections. In this context, the remaining case $\lambda_{1}=\lambda_{2}=\lambda_{3}=-1$ can be 
thought of as corresponding to three consecutive reflections with respect to the coordinate planes.

Note that in the special orthogonal case, when the rotation axis is determined, the problem is reduced to a two-dimensional rotation. Thus, in order to study the action on a generic nonzero vector in $\mathrm{x} \in \mathbb{R}^{3}$, it would be convenient to decompose into parallel and perpendicular to $\alpha$ components. We do so by introducing the projectors

$$
\mathcal{P}_{\alpha}^{\perp}=\hat{\mathbf{n}} \otimes \hat{\mathbf{n}}^{t}, \quad \mathcal{P}_{\alpha}=\mathcal{I}-\hat{\mathbf{n}} \otimes \hat{\mathbf{n}}^{t}
$$

where $\left(\hat{\mathbf{n}} \otimes \hat{\mathbf{n}}^{t}\right)_{i j}=\hat{n}_{i} \hat{n}_{j}$ stands for the usual dyadic (tensor) product of vectors. Then we have $\mathbf{x}=\mathbf{x}_{\alpha}+\mathbf{x}_{\alpha}^{\perp}$ with $\mathbf{x}_{\alpha}=\mathcal{P}_{\alpha} \mathbf{x}$ and $\mathbf{x}_{\alpha}^{\perp}=\mathcal{P}_{\alpha}^{\perp} \mathbf{x}$ respectively and the action of $\mathcal{R}$ is decomposed as well, since the orthogonal component remains unaltered, i.e.,

$$
\tilde{\mathbf{x}}=\mathcal{R} \mathbf{x}=\tilde{\mathbf{x}}_{\alpha}+\mathbf{x}_{\alpha}^{\perp}=\left(\mathcal{R}_{\alpha} \mathcal{P}_{\alpha}+\mathcal{P}_{\alpha}^{\perp}\right) \mathbf{x} .
$$

Here $\mathcal{R}_{\alpha}$ is the restriction of $\mathcal{R}$ to $\alpha$, which is a two-dimensional rotation, that in a suitably chosen basis can be simply expressed in terms of trigonometric functions of the angle $\varphi$. It requires no more than high school trigonometry to show that

$$
\mathcal{R}_{\alpha} \mathbf{x}_{\alpha}=\cos \varphi \mathbf{x}_{\alpha}+\sin \varphi \hat{\mathbf{n}} \times \mathbf{x}
$$

where the vector $\hat{\mathbf{n}} \times \mathbf{x}=\hat{\mathbf{n}} \times \mathbf{x}_{\alpha}$ actually completes the basis - it is normal to both $\hat{\mathbf{n}}$ and $\mathbf{x}_{\alpha}$, and the orientation agrees with the direction of rotation.

If we want to describe the action on $\mathbf{x}$ in operator form, it is convenient to use the Hodge duality between $\mathbb{R}^{3}$ vectors and skew-symmetric rank two tensors

$$
*: \mathbf{c} \rightarrow \mathbf{c}^{\times}, \quad \mathbf{c}^{\times} \mathbf{x}=\mathbf{c} \times \mathbf{x}, \quad \mathbf{x} \in \mathbb{R}^{3} .
$$

Using the symbol of Levi-Civita and Einstein convention for summation over repeated indices, we can easily write this in components as $\left(\mathbf{c}^{\times}\right)_{i j}=\varepsilon_{i l j} c_{l}$, or in coordinates

$$
\mathbf{c}^{\times}=\left(\begin{array}{rcc}
0 & -c_{3} & c_{2} \\
c_{3} & 0 & -c_{1} \\
-c_{2} & c_{1} & 0
\end{array}\right) .
$$

Combining the above results, we end up with

$$
\tilde{\mathbf{x}}=\mathcal{R} \mathbf{x}=\left(\mathcal{P}_{\alpha}^{\perp}+\cos \varphi \mathcal{P}_{\alpha}+\sin \varphi \hat{\mathbf{n}}^{\times}\right) \mathbf{x}
$$

and the expression in the brackets can be easily converted into the form

$$
\mathcal{R}(\mathbf{n}, \varphi)=\cos \varphi \mathcal{I}+(1-\cos \varphi) \hat{\mathbf{n}} \otimes \hat{\mathbf{n}}^{t}+\sin \varphi \hat{\mathbf{n}}^{\times}
$$


which is the famous Rodrigues' formula we use extensively in our construction.

Note that the above may be used in both directions: on the one hand, it determines the rotation as a function of $\hat{\mathbf{n}}$ and $\varphi$ while on the other, if $\mathcal{R}$ is explicitly given from empirical data or by the Gram-Schmidt procedure for example, one may easily derive from it both the axis $\hat{\mathbf{n}}$ and the angle $\varphi$.

For the axis it suffices to take the skew-symmetric part of the rotational matrix $\mathcal{A}=\frac{1}{2}\left(\mathcal{R}-\mathcal{R}^{t}\right)$, then use (2) to find the direction of $\hat{\mathbf{n}}$ and normalize. If $\varphi=\pi$ or close, $\sin \varphi$ is close to zero and it is either impossible or numerically incorrect to determine $\hat{\mathbf{n}}$ in that way. Instead, one may use that (3) reduces to

$$
\mathcal{O}(\hat{\mathbf{n}})=\mathcal{R}(\hat{\mathbf{n}}, \pi)=2 \hat{\mathbf{n}} \otimes \hat{\mathbf{n}}^{t}-\mathcal{I}
$$

for $\varphi=\pi$. Then, finding the components of $\hat{\mathbf{n}}$ is by no means more difficult, although in this case the vector is determined only up to a sign (as it should be, since rotating by an angle $\pi$ in the clockwise and counterclockwise direction is one and the same thing). We usually start with the diagonal elements, choose the component with greatest square, use it to divide the off-diagonal entries etc. If $\varphi$ is only close to 0 or $\pi$, one could work with the symmetric part $\mathcal{S}=\frac{1}{2}\left(\mathcal{R}+\mathcal{R}^{t}\right)$ instead, and use the fact that $\cos \varphi=\frac{1}{2}(\operatorname{Tr} \mathcal{R}-1)$. In order to determine $\varphi$ completely, one may retrieve $\sin \varphi$ again from the skew-symmetric part of $\mathcal{R}$, using the components of $\hat{\mathbf{n}}$.

Moreover, we could obtain another representation for $\mathcal{R}$ by using the well known trigonometric Euler substitution $\tau=\tan \frac{\varphi}{2}$. With its help (3) becomes

$$
\mathcal{R}(\hat{\mathbf{n}}, \varphi)=\frac{\left(1-\tau^{2}\right) \mathcal{I}+2 \tau^{2} \hat{\mathbf{n}} \otimes \hat{\mathbf{n}}^{t}+2 \tau \hat{\mathbf{n}}^{\times}}{1+\tau^{2}}
$$

and if we define the vector parameter of the rotation to be $\mathbf{c}=\tau \hat{\mathbf{n}}$, then we have the representation

$$
\mathcal{R}(\mathbf{c})=\frac{\left(1-\mathbf{c}^{2}\right) \mathcal{I}+2 \mathbf{c} \otimes \mathbf{c}^{t}+2 \mathbf{c}^{\times}}{1+\mathbf{c}^{2}}
$$

that can be written in coordinates as

$$
\mathcal{R}(\mathbf{c})=\frac{2}{1+\mathbf{c}^{2}}\left(\begin{array}{ccc}
1+c_{1}^{2} & c_{1} c_{2}-c_{3} & c_{1} c_{3}+c_{2} \\
c_{1} c_{2}+c_{3} & 1+c_{2}^{2} & c_{2} c_{3}-c_{1} \\
c_{3} c_{1}-c_{2} & c_{3} c_{2}+c_{1} & 1+c_{3}^{2}
\end{array}\right)-\mathcal{I} .
$$

Note that we no longer need to write two arguments - the angle is parameterized by $\tau$, which we refer to as scalar parameter and it is included in the definition of the vector parameter $\mathbf{c}$. 
There is a natural composition law for the vector parameters that comes quite handy for our considerations. Namely, from the group properties of the $\mathrm{SO}(3)$ matrices it can be derived that the vector parameter of the composition $\mathcal{R}(\mathbf{a}) \mathcal{R}(\mathbf{b})=$ $\mathcal{R}(\langle\mathbf{a}, \mathbf{b}\rangle)=\mathcal{R}(\mathbf{c})$ is

$$
\mathbf{c}=\langle\mathbf{a}, \mathbf{b}\rangle=\frac{\mathbf{a}+\mathbf{b}+\mathbf{a} \times \mathbf{b}}{1-(\mathbf{a}, \mathbf{b})} .
$$

In the above formula " $\times$ ", respectively $(\cdot, \cdot)$ denote the cross and dot products of vectors in $\mathbb{R}^{3}$. It is not so difficult to write a similar formula for the composition of three rotations. In that case it can be shown that the vector parameter of the rotation $\mathcal{R}(\mathbf{a}) \mathcal{R}(\mathbf{b}) \mathcal{R}(\mathbf{c})$ is

$$
\langle\langle\mathbf{a}, \mathbf{b}\rangle, \mathbf{c}\rangle=\frac{\mathbf{a}+\mathbf{b}+\mathbf{c}+\mathbf{a} \times \mathbf{b}+\mathbf{a} \times \mathbf{c}+\mathbf{b} \times \mathbf{c}+(\mathbf{a} \times \mathbf{b}) \times \mathbf{c}-(\mathbf{a}, \mathbf{b}) \mathbf{c}}{1-(\mathbf{a}, \mathbf{b})-(\mathbf{a}, \mathbf{c})-(\mathbf{b}, \mathbf{c})-(\mathbf{a}, \mathbf{b} \times \mathbf{c})} .
$$

Note that this operation is associative, but not commutative, unless the vectors are parallel - a property which is inherited from the group composition.

We refer to [7] for details regarding the derivation of (5) and (6).

It is worth mentioning in this context that the vector-parameter is also known as Gibbs' or Rodrigues' vector, while some authors call it "vector of finite rotations". The vector representation of rotations in three-dimensional space $\mathbb{R}^{3}$ is a principal subject of consideration in many papers and a far from exhaustive list includes [1], [9], [12], [18], [25], [32] and references therein. Actually, Fedorov [7] explores the vector-parametrization mainly in the context of problems related to the Lorentz group, while two of the present authors are among the first who make use of it in the classical [19] and quantum mechanics [15-17]. For more detailed discussion on the historical and mathematical aspects of Euler's theorem we refer to the paper by Palais et al [23], and for physical viewpoint on the subject - to the book by Goldstein [10].

The principal aim of the paper is to derive explicit formulas for the scalar parameters and the conditions under which certain decomposition is possible. These are given in analytical and easy to use form, which we hope will be appreciated by people, involved with practical applications of this problem. We left however aside the question about the topological nature behind them as this subject is discussed in depth in the papers by Stuelpnagel [31] and Perelyaev [24].

\section{The Generic Case}

Our starting data consist of the rotational matrix $\mathcal{R}$ and three nonzero vectors $\stackrel{\mathbf{c}}{k}_{k}, k=1,2,3$, possibly coplanar, but such that $\stackrel{\mathbf{c}}{2}_{2}$ is not parallel to any of the 
other two. After normalization, they will be used as rotational axes in our decomposition

$$
\mathcal{R}(\mathbf{c})=\mathcal{R}\left(\mathbf{c}_{3}\right) \mathcal{R}\left(\mathbf{c}_{2}\right) \mathcal{R}\left(\mathbf{c}_{1}\right)
$$

where $\mathbf{c}=\tau \hat{\mathbf{n}},|\hat{\mathbf{n}}|=1$ and $\tau=\tan \frac{\varphi}{2}$.

Our task is to find the scalar parameters $u, v, w$ (sometimes called also generalized Euler parameters) introduced via the relations

$$
\mathbf{c}_{1}=u \hat{\mathbf{c}}_{1}, \quad \mathbf{c}_{2}=v \hat{\mathbf{c}}_{2}, \quad \mathbf{c}_{3}=w \hat{\mathbf{c}}_{3} .
$$

In order to derive the values of $u, v$ and $w$ we use the defining relation (7) and suitably chosen scalar products as proposed in [20]. Here we start with

$$
\left(\hat{\mathbf{c}}_{3}, \mathcal{R}(\mathbf{c}) \hat{\mathbf{c}}_{1}\right)=\left(\hat{\mathbf{c}}_{3}, \mathcal{R}\left(w \hat{\mathbf{c}}_{3}\right) \mathcal{R}\left(v \hat{\mathbf{c}}_{2}\right) \mathcal{R}\left(u \hat{\mathbf{c}}_{1}\right) \hat{\mathbf{c}}_{1}\right)=\left(\hat{\mathbf{c}}_{3}, \mathcal{R}\left(v \hat{\mathbf{c}}_{2}\right) \hat{\mathbf{c}}_{1}\right)
$$

and make use of the fact that $\hat{\mathbf{c}}_{1}$ is an eigenvector of $\mathcal{R}\left(\mathbf{c}_{1}\right)$ with eigenvalue equal to one and so is $\hat{\mathbf{c}}_{3}$ for $\mathcal{R}\left(\mathbf{c}_{3}\right)$ independently of the values of their scalar parameters. In combination with (4) this leads to a quadratic equation for $v$ in the form

$$
\left(\sigma_{31}+\kappa_{31}-2 \kappa_{12} \kappa_{23}\right) v^{2}+2 \omega v+\sigma_{31}-\kappa_{31}=0
$$

in which for simplicity we have used the notations

$$
\kappa_{i j}=\left(\hat{\mathbf{c}}_{i}, \hat{\mathbf{c}}_{j}\right), \quad \sigma_{i j}=\left(\hat{\mathbf{c}}_{i}, \mathcal{R}(\mathbf{c}) \hat{\mathbf{c}}_{j}\right), \quad \omega=\left(\hat{\mathbf{c}}_{1}, \hat{\mathbf{c}}_{2} \times \hat{\mathbf{c}}_{3}\right) .
$$

This equation has real solutions whenever its discriminant is non-negative, i.e.,

$$
\omega^{2}=\operatorname{det}(\kappa) \geq \sigma_{31}^{2}-\kappa_{31}^{2}-2 \kappa_{12} \kappa_{23}\left(\sigma_{31}-\kappa_{31}\right) .
$$

Taking into account that $\kappa$ is symmetric with diagonal elements equal to one, we may easily write its determinant as

$$
\operatorname{det}(\kappa)=1+2 \kappa_{12} \kappa_{23} \kappa_{13}-\kappa_{12}^{2}-\kappa_{13}^{2}-\kappa_{23}^{2}
$$

which allows to express the above inequality as

$$
\Delta=\left|\begin{array}{ccc}
1 & \kappa_{12} & \sigma_{31} \\
\kappa_{21} & 1 & \kappa_{23} \\
\sigma_{31} & \kappa_{32} & 1
\end{array}\right| \geq 0 .
$$

Thus we recover the condition

$$
\left(\hat{\mathbf{c}}_{1},\left(\mathcal{R}(\mathbf{c})-\hat{\mathbf{c}}_{2} \otimes \hat{\mathbf{c}}_{2}^{t}\right) \hat{\mathbf{c}}_{3}\right)^{2} \leq\left(1-\left(\hat{\mathbf{c}}_{1}, \hat{\mathbf{c}}_{2}\right)^{2}\right)\left(1-\left(\hat{\mathbf{c}}_{3}, \hat{\mathbf{c}}_{2}\right)^{2}\right)
$$


given in [26]. The reader will find there also another derivation of the above formula and a detailed proof why it should be considered both necessary and sufficient condition (provided $\hat{\mathbf{c}}_{2}$ is not parallel to either $\hat{\mathbf{c}}_{1}$ or $\hat{\mathbf{c}}_{3}$ ) for the existence of the decomposition (7). We use it simply to test whether or not a certain choice of the axes is appropriate for the specified rotational matrix $\mathcal{R}$.

If the necessary condition is fulfilled, we may solve the above equation for $v$ and use the two roots $v_{ \pm}$for the computation of the corresponding matrix entries of $\mathcal{R}\left(\mathbf{c}_{1}\right)$ in the same way

$$
\left(\hat{\mathbf{c}}_{3}, \mathcal{R}(\mathbf{c}) \hat{\mathbf{c}}_{2}\right)=\left(\hat{\mathbf{c}}_{3}, \mathcal{R}\left(w \hat{\mathbf{c}}_{3}\right) \mathcal{R}\left(v_{ \pm} \hat{\mathbf{c}}_{2}\right) \mathcal{R}\left(u \hat{\mathbf{c}}_{1}\right) \hat{\mathbf{c}}_{2}\right)=\left(\hat{\mathbf{c}}_{3}, \mathcal{R}\left(v_{ \pm} \hat{\mathbf{c}}_{2}\right) \mathcal{R}\left(u \hat{\mathbf{c}}_{1}\right) \hat{\mathbf{c}}_{2}\right)
$$

and this leads to a pair of quadratic equations for the scalar parameter $u$

$$
\left(\left(\sigma_{32}+\kappa_{32}\right)\left(1+v_{ \pm}^{2}\right)+2 \kappa_{12} \nu_{ \pm}\right) u^{2}+2 \mu_{ \pm} u+\left(\sigma_{32}-\kappa_{32}\right)\left(1+v_{ \pm}^{2}\right)=0
$$

where

$$
\begin{aligned}
& \nu_{ \pm}=\kappa_{13}\left(v_{ \pm}^{2}-1\right)-2 \kappa_{12} \kappa_{23} v_{ \pm}^{2}+2 \omega v_{ \pm} \\
& \mu_{ \pm}=\omega\left(v_{ \pm}^{2}-1\right)+2\left(\kappa_{12} \kappa_{23}-\kappa_{13}\right) v_{ \pm} .
\end{aligned}
$$

Hence we have four solutions for $u$. Now it remains to determine the value of $w$ by exploiting

$$
\begin{aligned}
\left(\hat{\mathbf{c}}_{2}, \mathcal{R}(\mathbf{c}) \hat{\mathbf{c}}_{1}\right) & =\left(\hat{\mathbf{c}}_{2}, \mathcal{R}\left(w \hat{\mathbf{c}}_{3}\right) \mathcal{R}\left(v_{ \pm} \hat{\mathbf{c}}_{2}\right) \mathcal{R}\left(u \hat{\mathbf{c}}_{1}\right) \hat{\mathbf{c}}_{1}\right) \\
& =\left(\hat{\mathbf{c}}_{2}, \mathcal{R}\left(w \hat{\mathbf{c}}_{3}\right) \mathcal{R}\left(v_{ \pm} \hat{\mathbf{c}}_{2}\right) \hat{\mathbf{c}}_{1}\right)
\end{aligned}
$$

from which we derive the corresponding set of quadratic equations

$$
\left(\left(\sigma_{21}+\kappa_{21}\right)\left(v_{ \pm}^{2}+1\right)+2 \kappa_{23} \nu_{ \pm}\right) w^{2}+2 \mu_{ \pm} w+\left(\sigma_{21}-\kappa_{21}\right)\left(v_{ \pm}^{2}+1\right)=0 .
$$

In this way we end up with eight candidates for Euler decomposition, since each of the three quadratic equations has two solutions in the generic case. In order to choose the actual solution or solutions to the problem among these eight possibilities one may simply compare their matrix entries with the corresponding entries of the matrix $\mathcal{R}(\mathbf{c})$.

Alternatively, we may utilize the vector parameter composition

$$
\mathbf{c}=\left\langle\mathbf{c}_{3},\left\langle\mathbf{c}_{2}, \mathbf{c}_{1}\right\rangle\right\rangle
$$

or its equivalent forms

$$
\begin{aligned}
& \mathbf{c}_{1}=\left\langle-\mathbf{c}_{2},\left\langle-\mathbf{c}_{3}, \mathbf{c}\right\rangle\right\rangle \\
& \mathbf{c}_{2}=\left\langle-\mathbf{c}_{3},\left\langle\mathbf{c},-\mathbf{c}_{1}\right\rangle\right\rangle \\
& \mathbf{c}_{3}=\left\langle\mathbf{c},\left\langle-\mathbf{c}_{1},-\mathbf{c}_{2}\right\rangle\right\rangle
\end{aligned}
$$


which, in accordance with (6), can be expanded as

$$
\begin{aligned}
u \hat{\mathbf{c}}_{1} & =\frac{\tau \hat{\mathbf{n}}-v \hat{\mathbf{c}}_{2}-w \hat{\mathbf{c}}_{3}+v w \hat{\mathbf{c}}_{2} \times \hat{\mathbf{c}}_{3}+v \tau \hat{\mathbf{n}} \times \hat{\mathbf{c}}_{2}+w \tau \hat{\mathbf{n}} \times \hat{\mathbf{c}}_{3}+v w \tau \mathbf{m}_{1}}{1-v w \kappa_{23}+v \tau \rho_{2}+w \tau \rho_{3}-u w \tau \tilde{\rho}_{1}} \\
v \hat{\mathbf{c}}_{2} & =\frac{\tau \hat{\mathbf{n}}-u \hat{\mathbf{c}}_{1}-w \hat{\mathbf{c}}_{3}+u w \hat{\mathbf{c}}_{3} \times \hat{\mathbf{c}}_{1}+w \tau \hat{\mathbf{n}} \times \hat{\mathbf{c}}_{3}-u \tau \hat{\mathbf{n}} \times \hat{\mathbf{c}}_{1}+u w \tau \mathbf{m}_{2}}{1-u w \kappa_{13}+u \tau \rho_{1}+w \tau \rho_{3}+u w \tau \tilde{\rho}_{2}} \\
w \hat{\mathbf{c}}_{3} & =\frac{\tau \hat{\mathbf{n}}-u \hat{\mathbf{c}}_{1}-v \hat{\mathbf{c}}_{2}+u v \hat{\mathbf{c}}_{1} \times \hat{\mathbf{c}}_{2}-u \tau \hat{\mathbf{n}} \times \hat{\mathbf{c}}_{1}-v \tau \hat{\mathbf{n}} \times \hat{\mathbf{c}}_{2}+u v \tau \mathbf{m}_{3}}{1-u v \kappa_{12}+u \tau \rho_{1}+v \tau \rho_{2}-u v \tau \tilde{\rho}_{3}}
\end{aligned}
$$

In the above equations we have used the notations

$$
\rho_{k}=\left(\hat{\mathbf{c}}_{k}, \hat{\mathbf{n}}\right), \quad \varepsilon_{i j k} \tilde{\rho}_{k}=\left(\hat{\mathbf{c}}_{i} \times \hat{\mathbf{c}}_{j}, \hat{\mathbf{n}}\right)
$$

and

$$
\begin{aligned}
& \mathbf{m}_{1}=\rho_{2} \hat{\mathbf{c}}_{3}-\rho_{3} \hat{\mathbf{c}}_{2}-\kappa_{23} \hat{\mathbf{n}} \\
& \mathbf{m}_{2}=\kappa_{13} \hat{\mathbf{n}}-\rho_{1} \hat{\mathbf{c}}_{3}-\rho_{3} \hat{\mathbf{c}}_{1} \\
& \mathbf{m}_{3}=\rho_{2} \hat{\mathbf{c}}_{1}-\rho_{1} \hat{\mathbf{c}}_{2}-\kappa_{12} \hat{\mathbf{n}} .
\end{aligned}
$$

Multiplying both sides of the $k^{t h}$ equation in (12) with $\hat{\mathbf{c}}_{l}^{\times}$on the left, we obtain three systems of lower rank. Each one of them contains at least one nontrivial relation that can be extracted by considering its scalar product with $\hat{\mathbf{n}}$. In particular, from the first and the third one we easily derive expressions for $u(v, \tau)$ and $w(v, \tau)$, where $v$ is given by (16) as a solution to (8), more precisely these are

$$
u=\frac{\left(\kappa_{23}-\rho_{2} \rho_{3}\right) \tau v-\tilde{\rho}_{1} v}{\left(\rho_{1} \tilde{\rho}_{1}+\rho_{2} \tilde{\rho}_{2}\right) \tau v+\left(\kappa_{23} \rho_{1}-\kappa_{13} \rho_{2}\right) v+\left(\rho_{1} \rho_{3}-\kappa_{13}\right) \tau-\tilde{\rho}_{2}}
$$

and

$$
w=\frac{\left(\kappa_{12}-\rho_{1} \rho_{2}\right) \tau v-\tilde{\rho}_{3} v}{\left(\rho_{2} \tilde{\rho}_{2}+\rho_{3} \tilde{\rho}_{3}\right) \tau v+\left(\kappa_{12} \rho_{3}-\kappa_{13} \rho_{2}\right) v+\left(\rho_{1} \rho_{3}-\kappa_{13}\right) \tau-\tilde{\rho}_{2}} .
$$

From the second equation we obtain a similar expression for $w=w(u, \tau)$

$$
w=\frac{\left(\kappa_{12}-\rho_{1} \rho_{2}\right) \tau u-\tilde{\rho}_{3} u}{\left(\rho_{3} \tilde{\rho}_{3}-\rho_{1} \tilde{\rho}_{1}\right) \tau u+\left(\kappa_{12} \rho_{3}-\kappa_{23} \rho_{1}\right) u+\left(\kappa_{23}-\rho_{2} \rho_{3}\right) \tau-\tilde{\rho}_{1}} .
$$

However, the first two actually complete the solution after substituting the values of $v_{ \pm}$derived from (8)

$$
v_{ \pm}=\frac{-\omega \pm \sqrt{\Delta}}{\sigma_{31}+\kappa_{31}-2 \kappa_{12} \kappa_{23}}
$$

where $\Delta$ is defined in (9). 
As for the matrix element $\sigma_{31}$, we may easily express it from (3) as

$$
\sigma_{31}=\rho_{1} \rho_{3}+\left(\kappa_{13}-\rho_{1} \rho_{3}\right) \cos \varphi-\tilde{\rho}_{2} \sin \varphi
$$

that can be easily generalized as

$$
\sigma_{i j}=\rho_{i} \rho_{j}+\left(\kappa_{i j}-\rho_{i} \rho_{j}\right) \cos \varphi-\varepsilon_{i j k} \tilde{\rho}_{k} \sin \varphi .
$$

Note that for a fixed rotational angle and therefore scalar parameter $\tau$, the expressions (13), (14) and (15) are linear fractional functions that can be easily inverted, so we have analogous expressions for $u(w), v(u)$ and $v(w)$. These are certainly consistent with each other, so we may easily derive a quadratic equation for one of the parameters, similar to (8) and the other two will be expressed by it in a linear fractional manner.

If we consider the simple and yet interesting from practical point of view example in which the vectors $\hat{\mathbf{c}}_{k}$ coincide with the coordinate axes (Bryan decomposition). Applying the method described above we easily obtain the relations

$$
\left(\begin{array}{c}
u_{ \pm} \\
w_{ \pm}
\end{array}\right)=\frac{\tau}{1-\tau \hat{n}_{2} v_{ \pm}}\left(\begin{array}{l}
\hat{n}_{1}+\hat{n}_{3} v_{ \pm} \\
\hat{n}_{3}+\hat{n}_{1} v_{ \pm}
\end{array}\right)
$$

in which we have to substitute the solution to (8) given by the formula

$$
v_{ \pm}=\frac{-1 \pm \sqrt{1-\sigma_{31}^{2}}}{\sigma_{31}}
$$

In other words, the final result can be expressed in terms of explicit functions of $v_{ \pm}, \varphi$ and $\hat{\mathbf{n}}$.

In a similar way one may express $u_{ \pm}\left(v_{ \pm}, \mathbf{c}\right), w_{ \pm}\left(v_{ \pm}, \mathbf{c}\right)$ as linear fractional functions for the classical Euler decomposition setting in which the successive rotations are about the axes $O Z, O X$ and again $O Z$ in this specified order

$$
\left(\begin{array}{c}
u_{ \pm} \\
w_{ \pm}
\end{array}\right)=\frac{\tau}{\tau \hat{n}_{1}+v_{ \pm}}\left(\begin{array}{l}
\hat{n}_{3} v_{ \pm}-\hat{n}_{2} \\
\hat{n}_{3} v_{ \pm}+\hat{n}_{2}
\end{array}\right) .
$$

In this case the solutions to (8) are given by the formula

$$
v_{ \pm}= \pm \sqrt{\frac{1-\sigma_{31}}{1+\sigma_{31}}}
$$

Note that this approach is not directly applicable if the rotational matrix $\mathcal{R}(\mathbf{c})$ is a half turn since when $\tau \rightarrow \infty$ the denominator in (6) vanishes and this means that the case should be treated separately which is done in the section to follow. 


\section{The Symmetric Case}

Let us now consider the particular case of a nontrivial symmetric orthogonal matrix

$$
\mathcal{R}(\hat{\mathbf{n}}, \pi)=2 \hat{\mathbf{n}} \otimes \hat{\mathbf{n}}^{t}-\mathcal{I}=\mathcal{O}(\hat{\mathbf{n}})
$$

that represents a rotation by an angle $\pi$ about the axis, specified by the unit vector $\hat{\mathbf{n}}$. It is easy to see that $-\mathcal{O}(\mathbf{n})$ is a mirror reflection with respect to the plane, normal to $\hat{\mathbf{n}}$.

Applying directly the ideas from the previous section, we find that (8) can be written in this case as

$$
\left(\rho_{1} \rho_{3}-\kappa_{12} \kappa_{23}\right) v^{2}+\omega v+\rho_{1} \rho_{3}-\kappa_{13}=0
$$

with the obvious solution

$$
v_{ \pm}=\frac{-\omega \pm \sqrt{\Delta}}{2\left(\rho_{1} \rho_{3}-\kappa_{12} \kappa_{23}\right)} .
$$

The additional condition for the discriminant

$$
\omega^{2}=\operatorname{det}(\kappa) \geq 4\left(\rho_{1} \rho_{3}-\kappa_{12} \kappa_{23}\right)\left(\rho_{1} \rho_{3}-\kappa_{13}\right)
$$

in this case can be written as

$$
4\left(\rho_{1} \rho_{3}-\kappa_{12} \kappa_{23}-\kappa_{13}\right) \rho_{1} \rho_{3} \leq 1-2 \kappa_{12} \kappa_{23} \kappa_{13}-\kappa_{12}^{2}-\kappa_{13}^{2}-\kappa_{23}^{2} .
$$

Similarly from (10) and (11) we get

$$
\left(\rho_{2} \rho_{3}\left(v_{ \pm}^{2}+1\right)+\kappa_{12} \nu_{ \pm}\right) u^{2}+\mu_{ \pm} u+\left(\rho_{2} \rho_{3}-\kappa_{23}\right)\left(v_{ \pm}^{2}+1\right)=0
$$

which gives the possible values of $u$, while for $w$ they can be specified from the equation

$$
\left(\rho_{1} \rho_{2}\left(v_{ \pm}^{2}+1\right)+\kappa_{23} \nu_{ \pm}\right) w^{2}+\mu_{ \pm} w+\left(\rho_{1} \rho_{2}-\kappa_{12}\right)\left(v_{ \pm}^{2}+1\right)=0
$$

All considerations in the previous section are applicable here, except for the relations, derived from the composition law for the vector parameters, because the denominator of (6) vanishes in the symmetric case. This can be concluded directly from (4). One way to make $\mathcal{R}(\mathbf{c})$ symmetric is to set the scalar parameter equal to zero, but then one gets the identity matrix. The other possibility is to take the limit $|\mathbf{c}| \rightarrow \infty$ in (4), which is the only way to obtain a half turn of the type (18). Since this limiting procedure yields a vanishing denominator for the composition of the vector parameters in (6), we have

$$
\left(\mathbf{c}_{1}, \mathbf{c}_{2}\right)+\left(\mathbf{c}_{1}, \mathbf{c}_{3}\right)+\left(\mathbf{c}_{2}, \mathbf{c}_{3}\right)-\left(\mathbf{c}_{1}, \mathbf{c}_{2} \times \mathbf{c}_{3}\right)=1
$$


In terms of the scalar parameters it can be rewritten as

$$
\kappa_{12} u v+\kappa_{13} u w+\kappa_{23} v w-\omega u v w=1 .
$$

On the other hand, once we have the value of $v$ fixed from (19), we may use directly the results (13) and (14) in the limit $\tau \rightarrow \infty$ in order to obtain

$$
\begin{aligned}
u(v) & =\frac{\left(\kappa_{23}-\rho_{2} \rho_{3}\right) v}{\left(\rho_{1} \tilde{\rho}_{1}+\rho_{2} \tilde{\rho}_{2}\right) v+\rho_{1} \rho_{3}-\kappa_{13}} \\
w(v) & =\frac{\left(\kappa_{12}-\rho_{1} \rho_{2}\right) v}{\left(\rho_{2} \tilde{\rho}_{2}+\rho_{3} \tilde{\rho}_{3}\right) v+\rho_{1} \rho_{3}-\kappa_{13}}
\end{aligned}
$$

which concludes the solution in the symmetric case.

Actually, it is possible to obtain equation (19) by the same construction, so it is sufficient to resolve the symmetric case. Note also that in general we can express any two of the scalar parameters as a function of the complementary one just as in equations (23), i.e.,

$$
\begin{aligned}
& u(w)=\frac{\left(\rho_{2} \rho_{3}-\kappa_{23}\right) w}{\left(\rho_{3} \tilde{\rho}_{3}-\rho_{1} \tilde{\rho}_{1}\right) w+\rho_{1} \rho_{2}-\kappa_{12}} \\
& v(w)=\frac{\left(\kappa_{13}-\rho_{1} \rho_{3}\right) w}{\left(\rho_{2} \tilde{\rho}_{2}+\rho_{3} \tilde{\rho}_{3}\right) w+\rho_{1} \rho_{2}-\kappa_{12}}
\end{aligned}
$$

and finally

$$
\begin{aligned}
v(u) & =\frac{\left(\kappa_{13}-\rho_{1} \rho_{3}\right) u}{\left(\rho_{1} \tilde{\rho}_{1}+\rho_{2} \tilde{\rho}_{2}\right) u+\rho_{2} \rho_{3}-\kappa_{23}} \\
w(u) & =\frac{\left(\rho_{1} \rho_{2}-\kappa_{12}\right) u}{\left(\rho_{1} \tilde{\rho}_{1}-\rho_{3} \tilde{\rho}_{3}\right) v+\rho_{2} \rho_{3}-\kappa_{23}}
\end{aligned}
$$

and this comes quite handy for our further considerations.

We conclude this section with the examples from the previous one, this time written for half turns. For the Bryan decomposition when the axes of rotation coincide with the coordinate axes, we easily find the solutions to be

$$
\left(\begin{array}{c}
u_{ \pm} \\
w_{ \pm}
\end{array}\right)=-\frac{1}{\hat{n}_{2} v_{ \pm}}\left(\begin{array}{l}
\hat{n}_{3} v_{ \pm}+\hat{n}_{1} \\
\hat{n}_{1} v_{ \pm}+\hat{n}_{3}
\end{array}\right)
$$

while in the Euler case we have

$$
\left(\begin{array}{l}
u_{ \pm} \\
w_{ \pm}
\end{array}\right)=\frac{1}{\hat{n}_{1}}\left(\begin{array}{l}
\hat{n}_{3} v_{ \pm}-\hat{n}_{2} \\
\hat{n}_{3} v_{ \pm}+\hat{n}_{2}
\end{array}\right) .
$$


Furthermore, one needs to substitute the solutions to (8) in both cases - the former being

$$
v_{ \pm}=\frac{-1 \pm \sqrt{1-4 \hat{n}_{1}^{2} \hat{n}_{3}^{2}}}{2 \hat{n}_{1} \hat{n}_{3}}
$$

while for the latter we have

$$
v_{ \pm}= \pm \frac{\sqrt{\hat{n}_{1}^{2}+\hat{n}_{2}^{2}}}{\hat{n}_{3}} .
$$

One may easily verify that in the first case we have, $u v w=-1$, as promised by formula (22), while in the second one, the relation is $u w=1$.

The cases, in which the denominators vanish, correspond to either decomposing into two rotations, or half turns, appearing in the decomposition. These require slightly different techniques, developed in the following two sections.

\section{Decomposition Into Two Rotations}

There is one more question that needs to be discussed in the present context. Namely, in certain cases one may ask if it is possible to write the vector decomposition for two, rather than three, rotational matrices, i.e.,

$$
\mathcal{R}(\mathbf{c})=\mathcal{R}\left(v \hat{\mathbf{c}}_{2}\right) \mathcal{R}\left(u \hat{\mathbf{c}}_{1}\right) .
$$

Then we need to specify only two parameters, but have less relations to work with. Generally speaking, it is not clear whether such a representation is possible at all, and a consistent treatment of the problem should clarify what conditions have to be fulfilled in order to alow it. If we apply the same approach as we did in the generic case, we end up with much simplified relations for the parameters

$$
\begin{aligned}
& \left(\hat{\mathbf{c}}_{2}, \mathcal{R}(\mathbf{c}) \hat{\mathbf{c}}_{1}\right)=\left(\hat{\mathbf{c}}_{2}, \mathcal{R}\left(v \hat{\mathbf{c}}_{2}\right) \mathcal{R}\left(u \hat{\mathbf{c}}_{1}\right) \hat{\mathbf{c}}_{1}\right)=\left(\hat{\mathbf{c}}_{2}, \hat{\mathbf{c}}_{1}\right) \\
& \left(\hat{\mathbf{c}}_{2}, \mathcal{R}(\mathbf{c}) \hat{\mathbf{c}}_{2}\right)=\left(\hat{\mathbf{c}}_{2}, \mathcal{R}\left(v \hat{\mathbf{c}}_{2}\right) \mathcal{R}\left(u \hat{\mathbf{c}}_{1}\right) \hat{\mathbf{c}}_{2}\right)=\left(\hat{\mathbf{c}}_{2}, \mathcal{R}\left(u \hat{\mathbf{c}}_{1}\right) \hat{\mathbf{c}}_{2}\right) \\
& \left(\hat{\mathbf{c}}_{1}, \mathcal{R}(\mathbf{c}) \hat{\mathbf{c}}_{1}\right)=\left(\hat{\mathbf{c}}_{1}, \mathcal{R}\left(v \hat{\mathbf{c}}_{2}\right) \mathcal{R}\left(u \hat{\mathbf{c}}_{1}\right) \hat{\mathbf{c}}_{1}\right)=\left(\hat{\mathbf{c}}_{1}, \mathcal{R}\left(v \hat{\mathbf{c}}_{2}\right) \hat{\mathbf{c}}_{1}\right) .
\end{aligned}
$$

From the first equation we have

$$
\sigma_{21}=\kappa_{21}
$$

which can be considered as a necessary condition for the existence of decomposition (26). In [26] it is shown that this condition is also sufficient, provided that $\kappa_{12} \neq \pm 1$, as in this case we have one, rather than two axes. 
From the second and the third relation we easily get the solutions for the scalar parameters $u$ and $v$ in the form

$$
\begin{aligned}
& u_{ \pm}= \pm \sqrt{\frac{1-\sigma_{22}}{1+\sigma_{22}-2 \kappa_{12}^{2}}}= \pm \frac{\sin \frac{\theta_{2}}{2}}{\sqrt{\cos ^{2} \frac{\theta_{2}}{2}-\cos ^{2} \gamma_{12}}} \\
& v_{ \pm}= \pm \sqrt{\frac{1-\sigma_{11}}{1+\sigma_{11}-2 \kappa_{12}^{2}}}= \pm \frac{\sin \frac{\theta_{1}}{2}}{\sqrt{\cos ^{2} \frac{\theta_{1}}{2}-\cos ^{2} \gamma_{12}}}
\end{aligned}
$$

where $\gamma_{12}$ is the angle between $\hat{\mathbf{c}}_{1}$ and $\hat{\mathbf{c}}_{2}$, and $\theta_{k}$ - that one by which $\mathcal{R}(\mathbf{c})$ rotates the vector $\hat{\mathbf{c}}_{k}$. A simple geometrical argument shows that the denominators vanish only if $\mathbf{c} \perp \hat{\mathbf{c}}_{1,2}$, which needs to be discussed separately. Note that in the particular case of perpendicular axes $\left(\kappa_{12}=0\right)$ we have

$$
u_{ \pm}= \pm \tan \frac{\theta_{2}}{2}, \quad v_{ \pm}= \pm \tan \frac{\theta_{1}}{2}
$$

which leads to $\left|\psi_{1}\right|=\left|\theta_{2}\right|, \quad\left|\psi_{2}\right|=\left|\theta_{1}\right|$ and the connection between the angles $\psi_{k}$ and $\varphi$ becomes apparent from Rodrigues' formula (3), since after taking an appropriate scalar products one ends up with

$$
\cos \theta_{k}=\cos \varphi+(1-\cos \varphi) \cos ^{2} \beta_{k}, \quad \beta_{k}=\measuredangle\left(\hat{\mathbf{n}}, \hat{\mathbf{c}}_{k}\right) .
$$

Also note that (28) demands an additional pair of conditions

$$
1+\sigma_{k k} \geq 2 \kappa_{21}^{2}, \quad k=1,2
$$

to be fulfilled in order to guarantee real solutions. These inequalities always hold for a simple geometrical reason: the maximal value of $\theta_{k}$ is obtained when $\hat{\mathbf{n}}$ is perpendicular to the plane, determined by the two rotational axes. Then $\mathcal{R}(\mathbf{c})$ is decomposed into a pair of reflections and the resulting angle equals in absolute value twice the angle between the two axes, that is $\left|\theta_{k}\right|=2 \min (|\gamma|,|\pi-\gamma|)$, $k=1,2$.

Next, we use the two vector compositions, we obtain from (26)

$$
\left\langle\mathbf{c},-\mathbf{c}_{1}\right\rangle=\mathbf{c}_{2}, \quad\left\langle-\mathbf{c}_{2}, \mathbf{c}\right\rangle=\mathbf{c}_{1}
$$

which produce directly

$$
u=\frac{\xi_{1}-\kappa_{12}\left(1+\xi_{1}\right) \xi_{2}}{1-\kappa_{12}^{2}\left(1-\xi_{1}\right)\left(1-\xi_{2}\right)}, \quad v=\frac{\xi_{2}-\kappa_{12}\left(1+\xi_{2}\right) \xi_{1}}{1-\kappa_{12}^{2}\left(1-\xi_{1}\right)\left(1-\xi_{2}\right)} .
$$


The easiest solution once more appears to be the case of perpendicular axes $\kappa_{12}=0$. Then we have $u=\xi_{1}$ and $v=\xi_{2}$. Since these solutions are bound to coincide with the obtained above, we have

$$
\tan \frac{\theta_{2}}{2}= \pm \tan \frac{\varphi}{2} \cos \beta_{1}, \quad \tan \frac{\theta_{1}}{2}= \pm \tan \frac{\varphi}{2} \cos \beta_{2} .
$$

Here we present another set of equivalent formulae, obtained by the method, presented at the end of Section 2. First we note that equation (17) can be cast in the form

$$
\sigma_{i j}-\kappa_{i j}=\left(\rho_{i} \rho_{j}-\kappa_{i j}\right)(1-\cos \varphi)-\varepsilon_{i j k} \tilde{\rho}_{k} \sin \varphi
$$

leading to the expressions for $\tau$ in the cases of two axes decomposition

$$
\sigma_{i j}=\kappa_{i j} \Longrightarrow\left(\rho_{i} \rho_{j}-\kappa_{i j}\right) \tau=\varepsilon_{i j k} \tilde{\rho}_{k} .
$$

Obviously, their explicit forms are given by the formulas

$$
\begin{gathered}
\sigma_{21}=\kappa_{21} \Rightarrow \tau=\frac{\tilde{\rho}_{3}}{\kappa_{12}-\rho_{1} \rho_{2}} \\
\sigma_{32}=\kappa_{32} \Rightarrow \tau=\frac{\tilde{\rho}_{1}}{\kappa_{23}-\rho_{2} \rho_{3}} \\
\sigma_{31}=\kappa_{31} \Rightarrow \tau=\frac{\tilde{\rho}_{2}}{\rho_{1} \rho_{3}-\kappa_{13}} .
\end{gathered}
$$

The rest of the procedure is basically considering the limit $w \rightarrow 0$ in the case we rotate about the first and the second axis, and similarly for the other two parameters. Note that (30) guarantees that the limit will always be of the type $\frac{0}{0}$, so we may use l'Hôspital's rule to obtain that when $w \rightarrow 0$ (or $w \rightarrow \infty$ ) is applied to (13), (14) and (15) produce the following expressions

$$
\begin{aligned}
& u=\frac{\left(\rho_{2} \rho_{3}-\kappa_{23}\right) \tau+\tilde{\rho}_{1}}{\left(\rho_{3} \tilde{\rho}_{3}-\rho_{1} \tilde{\rho}_{1}\right) \tau+\kappa_{12} \rho_{3}-\kappa_{23} \rho_{1}} \\
& v=\frac{\left(\kappa_{13}-\rho_{1} \rho_{3}\right) \tau+\tilde{\rho}_{2}}{\left(\rho_{2} \tilde{\rho}_{2}+\rho_{3} \tilde{\rho}_{3}\right) \tau+\kappa_{12} \rho_{3}-\kappa_{13} \rho_{2}} .
\end{aligned}
$$

On the same footing when $v \rightarrow 0, \infty$ we have additionally

$$
\begin{gathered}
u=\frac{\left(\kappa_{23}-\rho_{2} \rho_{3}\right) \tau-\tilde{\rho}_{1}}{\left(\rho_{1} \tilde{\rho}_{1}+\rho_{2} \tilde{\rho}_{2}\right) \tau+\kappa_{23} \rho_{1}-\kappa_{13} \rho_{2}} \\
w=\frac{\left(\kappa_{12}-\rho_{1} \rho_{2}\right) \tau-\tilde{\rho}_{3}}{\left(\rho_{2} \tilde{\rho}_{2}+\rho_{3} \tilde{\rho}_{3}\right) \tau+\kappa_{12} \rho_{3}-\kappa_{13} \rho_{2}}
\end{gathered}
$$


and finally for $u \rightarrow 0, \infty$

$$
\begin{aligned}
v & =\frac{\left(\kappa_{13}-\rho_{1} \rho_{3}\right) \tau+\tilde{\rho}_{2}}{\left(\rho_{1} \tilde{\rho}_{1}+\rho_{2} \tilde{\rho}_{2}\right) \tau+\kappa_{23} \rho_{1}-\kappa_{13} \rho_{2}} \\
w & =\frac{\left(\kappa_{12}-\rho_{1} \rho_{2}\right) \tau-\tilde{\rho}_{3}}{\left(\rho_{3} \tilde{\rho}_{3}-\rho_{1} \tilde{\rho}_{1}\right) \tau+\kappa_{12} \rho_{3}-\kappa_{23} \rho_{1}} .
\end{aligned}
$$

If, on the other hand, $\mathcal{R}(\mathbf{c})$ is a half turn itself

$$
\mathcal{O}(\hat{\mathbf{n}})=\mathcal{R}\left(v \hat{\mathbf{c}}_{2}\right) \mathcal{R}\left(u \hat{\mathbf{c}}_{1}\right)
$$

we may use (12) and then explore it in the limit $\tau \rightarrow \infty$. In this case

$$
\rho_{1} \rho_{2}-\kappa_{12}=0
$$

so we may use once more l'Hôpital's rule in order to obtain

$$
u=\frac{\rho_{2} \rho_{3}-\kappa_{23}}{\rho_{3} \tilde{\rho}_{3}-\rho_{1} \tilde{\rho}_{1}}, \quad v=\frac{\kappa_{13}-\rho_{1} \rho_{3}}{\rho_{2} \tilde{\rho}_{2}+\rho_{3} \tilde{\rho}_{3}} .
$$

If the rotations are about the first and the third axis, and the condition $\kappa_{13}=\rho_{1} \rho_{3}$ holds, we can proceed in the same way by using directly (23) for the case $v \rightarrow 0$ to end up with

$$
u=\frac{\kappa_{23}-\rho_{2} \rho_{3}}{\rho_{1} \tilde{\rho}_{1}+\rho_{2} \tilde{\rho}_{2}}, \quad w=\frac{\kappa_{12}-\rho_{1} \rho_{2}}{\rho_{2} \tilde{\rho}_{2}+\rho_{3} \tilde{\rho}_{3}} .
$$

Finally, decomposition about the second and the third axis demands that the condition $\kappa_{23}=\rho_{2} \rho_{3}$ is fulfilled and via (25) and l'Hôpital's rule we obtain

$$
v=\frac{\kappa_{13}-\rho_{1} \rho_{3}}{\rho_{1} \tilde{\rho}_{1}+\rho_{2} \tilde{\rho}_{2}}, \quad w=\frac{\rho_{1} \rho_{2}-\kappa_{12}}{\rho_{1} \tilde{\rho}_{1}-\rho_{3} \tilde{\rho}_{3}} .
$$

Let us briefly discuss the problem of vanishing denominators in equations (28). This phenomenon is quite natural to appear, since the scalar parameters are allowed to take infinite values in the symmetric case. In particular $\mathcal{R}(\mathbf{c})$ can be symmetric as well - for example a half turn about $O Z$ that is being decomposed into a product of two half turns (about $O X$ and $O Y$ respectively), or two mirror reflections with respect to perpendicular planes, as basic geometry teaches us. We can still come up with a solution - whenever $\sigma_{11}+1=2 \kappa_{12}^{2}$ for example, it is clear that $\psi_{2}=\pi$ and although the corresponding scalar parameter diverges, we may construct the matrix $\mathcal{R}\left(\mathbf{c}_{2}\right)$ based on (18). Cases like this are considered into more detail in the next section. 


\section{Cases Involving a Half Turn}

As has been thoroughly discussed above, the decomposition of a half turn into two or three rotations is not much different compared to the decomposition of a generic orthogonal matrix, especially on the level of quadratic equations. On the contrary, if a half turn takes part in the decomposition, the picture changes drastically due to the divergent scalar parameter. In this section we present several simple tricks to recognize and deal with the presence of one or more half turns in the decomposition.

Our strategy here is rather simple. We study the asymptotic behavior of the solutions (13), (14) and (15) as the corresponding parameter tends to infinity. The solutions tend to be as in the case of decomposition by two rotations, so we may take advantage of (32), (33) and (34) in the generic case and respectively (36), (37), (38) in the symmetric one.

Similarly, for two half turns we consider the double limit asymptotic and for this purpose we use a slightly different technique. Namely, instead of multiplying the equations in (12) by $\hat{\mathbf{c}}_{k}^{\times}$we take the scalar products with $\hat{\mathbf{c}}_{k}$ and in this way we obtain the dependance of each parameter on the remaining two

$$
\begin{aligned}
& u(v, w)=\frac{\rho_{1} \tau-\kappa_{12} v-\kappa_{13} w+\omega v w-\tilde{\rho}_{3} v \tau+\tilde{\rho}_{2} w \tau-\tilde{m} v w \tau}{1-\kappa_{23} v w+\rho_{2} v \tau+\rho_{3} w \tau-\tilde{\rho}_{1} v w \tau} \\
& v(u, w)=\frac{\rho_{2} \tau-\kappa_{12} u-\kappa_{23} w+\omega u w-\tilde{\rho}_{3} u \tau-\tilde{\rho}_{1} w \tau-\tilde{m} u w \tau}{1-\kappa_{13} u w+\rho_{1} u \tau+\rho_{3} w \tau+\tilde{\rho}_{2} u w \tau} \\
& w(u, v)=\frac{\rho_{3} \tau-\kappa_{13} u-\kappa_{23} v+\omega u v+\tilde{\rho}_{2} u \tau-\tilde{\rho}_{1} v \tau-\tilde{m} u v \tau}{1-\kappa_{12} u v+\rho_{1} u \tau+\rho_{2} v \tau-\tilde{\rho}_{3} u v \tau}
\end{aligned}
$$

where

$$
\tilde{m}=\kappa_{12} \rho_{3}+\kappa_{23} \rho_{1}-\kappa_{13} \rho_{2} .
$$

As a consequence we have the relations

$$
\begin{aligned}
\lim _{v, w \rightarrow \infty} u(v, w) & =\frac{\tilde{m} \tau-\omega}{\tilde{\rho}_{1} \tau+\kappa_{23}} \\
\lim _{u, w \rightarrow \infty} v(u, w) & =\frac{\tilde{m} \tau-\omega}{\kappa_{13}-\tilde{\rho}_{2} \tau} \\
\lim _{u, v \rightarrow \infty} w(u, v) & =\frac{\tilde{m} \tau-\omega}{\tilde{\rho}_{3} \tau+\kappa_{12}} .
\end{aligned}
$$


For the symmetric case we consider the limits of the above formulas when $\tau \rightarrow \infty$ and obtain a remarkably simple solution to the problem

$$
u=\frac{\tilde{m}}{\tilde{\rho}_{1}}, \quad v=-\frac{\tilde{m}}{\tilde{\rho}_{2}}, \quad w=\frac{\tilde{m}}{\tilde{\rho}_{3}} .
$$

The above formulae, together with their asymptotic behavior, practically cover all non-degenerate cases. In the following we discuss some geometrical arguments concerning the conditions under which certain decomposition involves a half turn.

\subsection{The Case of Two Rotations}

First we consider the case of two rotations as described in the previous section. As mentioned above, the most singular case is when the rotation matrix $\mathcal{R}(\mathbf{c})$ is itself a half turn and has to be decomposed into a pair of half turns about the axes $\hat{\mathbf{c}}_{1}$ and $\hat{\mathbf{c}}_{2}$ respectively. This is the same as a decomposition into a pair of reflections, which is only possible if the three axes are perpendicular to each other

$$
\rho_{1}=\rho_{2}=\kappa_{12}=0 \text {. }
$$

More generally, a rotation $\mathcal{R}(\mathbf{c})$ is decomposed into a pair of half turns if its axis $\hat{\mathbf{n}}$ is perpendicular to the plane determined by the vectors $\hat{\mathbf{c}}_{1}$ and $\hat{\mathbf{c}}_{2}$, and the angle $\varphi$ is equal to twice the angle between the axes. This may be written as

$$
\rho_{1}=\rho_{2}=0, \quad \kappa_{12}^{2}=\frac{1}{1+\tau^{2}} .
$$

Another option is to decompose a half turn into a generic rotation and another half turn

$$
\mathcal{O}(\hat{\mathbf{n}})=\mathcal{O}\left(\hat{\mathbf{c}}_{2}\right) \mathcal{R}\left(u \hat{\mathbf{c}}_{1}\right) .
$$

Multiplying both sides of the above equation on the left with $\mathcal{O}\left(\hat{\mathbf{c}}_{2}\right)$, we end up with the previous situation - decomposition of $\mathcal{R}\left(\mathbf{c}_{1}\right)$ into a pair of half turns. The orthogonality condition demands

$$
\kappa_{12}=\rho_{1}=0
$$

which can be used as a test, and if it is fulfilled, we have for the vector parameter $\mathbf{c}_{1}=\left\langle\mathbf{c}_{2}, \mathbf{c}\right\rangle$ which, considered in the limit $v, \tau \rightarrow \infty$, yields

$$
\mathbf{c}_{1}=\frac{\hat{\mathbf{n}} \times \hat{\mathbf{c}}_{2}}{\left(\hat{\mathbf{n}}, \hat{\mathbf{c}}_{2}\right)} .
$$


The value of the scalar parameter is fixed by the defining identity

$$
u=\left(\hat{\mathbf{c}}_{1}, \mathbf{c}_{1}\right)=\frac{\left(\hat{\mathbf{c}}_{1}, \hat{\mathbf{n}} \times \hat{\mathbf{c}}_{2}\right)}{\left(\hat{\mathbf{n}}, \hat{\mathbf{c}}_{2}\right)}=-\frac{\tilde{\rho}_{3}}{\rho_{2}} .
$$

A more direct way to obtain the same result is to set $w=0$ and let $v, \tau \rightarrow \infty$ in (39).

In particular, if we have $\rho_{2}=0$ in addition to the orthogonality conditions, we may conclude that $\mathcal{R}\left(\mathbf{c}_{1}\right)$ is also a half turn. Obviously, the case in which the factors on the right hand side are exchanged, i.e.,

$$
\mathcal{O}(\hat{\mathbf{n}})=\mathcal{R}\left(v \hat{\mathbf{c}}_{2}\right) \mathcal{O}\left(\hat{\mathbf{c}}_{1}\right)
$$

is quite similar and can be treated in the same way. This time the test produces the relations

$$
\kappa_{12}=\rho_{2}=0
$$

and the actual solution can be derived from the equation

$$
\mathbf{c}_{2}=\frac{\hat{\mathbf{c}}_{1} \times \hat{\mathbf{n}}}{\left(\hat{\mathbf{n}}, \hat{\mathbf{c}}_{1}\right)} .
$$

Therefore, for the scalar parameter we have the formula

$$
v=\left(\hat{\mathbf{c}}_{2}, \mathbf{c}_{2}\right)=\frac{\left(\hat{\mathbf{c}}_{2}, \hat{\mathbf{c}}_{1} \times \hat{\mathbf{n}}\right)}{\left(\hat{\mathbf{n}}, \hat{\mathbf{c}}_{1}\right)}=-\frac{\tilde{\rho}_{3}}{\rho_{1}}
$$

which can be obtained also from (39) at $w \rightarrow 0$, in the limit $u, \tau \rightarrow \infty$.

Finally, let us consider the case

$$
\mathcal{R}(\mathbf{c})=\mathcal{O}\left(\hat{\mathbf{c}}_{2}\right) \mathcal{R}\left(u \hat{\mathbf{c}}_{1}\right) .
$$

Choosing a suitable scalar product, one easily recovers the condition of vanishing denominator in the first relation of (28)

$$
2 \kappa_{12}^{2}=1+\sigma_{11}
$$

which is quite convenient to use as a test.

If the above condition is satisfied, we easily find

$$
u=-\frac{1}{\xi_{1}}=\frac{\rho_{1} \rho_{2}-\kappa_{12}}{\rho_{1} \tilde{\rho}_{3}}
$$

using the condition that ensures the vanishing of the denominator of composition vector $\left\langle\mathbf{c},-\mathbf{c}_{1}\right\rangle$ and the first relation in (31). 
In the case

$$
\mathcal{R}(\mathbf{c})=\mathcal{R}\left(v \hat{\mathbf{c}}_{2}\right) \mathcal{O}\left(\hat{\mathbf{c}}_{1}\right)
$$

analogous considerations lead to the formula

$$
v=-\frac{1}{\xi_{2}}=\frac{\rho_{1} \rho_{2}-\kappa_{12}}{\rho_{2} \tilde{\rho}_{3}}
$$

as long as the condition

$$
2 \kappa_{12}^{2}=1+\sigma_{22}
$$

is fulfilled.

Table 1. Two axes decompositions.

\begin{tabular}{|l|c|c|c|}
\hline \multicolumn{1}{|c|}{ Decomposition } & Criteria & $u$ & $v$ \\
\hline $\mathcal{R}(\mathbf{c})=\mathcal{R}\left(v \hat{\mathbf{c}}_{2}\right) \mathcal{R}\left(u \hat{\mathbf{c}}_{1}\right)$ & $(27)$ & $(29)$ & $(29)$ \\
\hline $\mathcal{R}(\mathbf{c})=\mathcal{O}\left(\hat{\mathbf{c}}_{2}\right) \mathcal{R}\left(u \hat{\mathbf{c}}_{1}\right)$ & $(27),(48)$ & $(49)$ & $\infty$ \\
\hline $\mathcal{R}(\mathbf{c})=\mathcal{R}\left(v \hat{\mathbf{c}}_{2}\right) \mathcal{O}\left(\hat{\mathbf{c}}_{1}\right)$ & $(27),(51)$ & $\infty$ & $(50)$ \\
\hline $\mathcal{R}(\mathbf{c})=\mathcal{O}\left(\hat{\mathbf{c}}_{2}\right) \mathcal{O}\left(\hat{\mathbf{c}}_{1}\right)$ & $(43)$ & $\infty$ & $\infty$ \\
\hline $\mathcal{O}(\hat{\mathbf{n}})=\mathcal{R}\left(v \hat{\mathbf{c}}_{2}\right) \mathcal{R}\left(u \hat{\mathbf{c}}_{1}\right)$ & $(35)$ & $(36)$ & $(36)$ \\
\hline $\mathcal{O}(\hat{\mathbf{n}})=\mathcal{O}\left(\hat{\mathbf{c}}_{2}\right) \mathcal{R}\left(u \hat{\mathbf{c}}_{1}\right)$ & $(44)$ & $(45)$ & $\infty$ \\
\hline $\mathcal{O}(\hat{\mathbf{n}})=\mathcal{R}\left(v \hat{\mathbf{c}}_{2}\right) \mathcal{O}\left(\hat{\mathbf{c}}_{1}\right)$ & $(46)$ & $\infty$ & $(47)$ \\
\hline $\mathcal{O}(\hat{\mathbf{n}})=\mathcal{O}\left(\hat{\mathbf{c}}_{2}\right) \mathcal{O}\left(\hat{\mathbf{c}}_{1}\right)$ & $(42)$ & $\infty$ & $\infty$ \\
\hline
\end{tabular}

Exploiting the same technique, we easily obtain the solutions for the decompositions into rotations about the first and the third, respectively the second and the third axes. The above formulae remain valid for $u, w$, respectively $v, w$ with only a slight adjustment of the indices.

All cases of two axes decompositions of rotations, along with the precise conditions under which they are justified, are presented in Table 1. 


\subsection{The Case of Three Rotations}

Now we focus on the generic case. To begin with, if $\mathcal{R}(\mathbf{c})$ is decomposed as

$$
\mathcal{R}(\mathbf{c})=\mathcal{O}\left(\hat{\mathbf{c}}_{3}\right) \mathcal{R}\left(v \hat{\mathbf{c}}_{2}\right) \mathcal{R}\left(u \hat{\mathbf{c}}_{1}\right)
$$

it would be convenient to use the fact that $\mathcal{O}\left(\hat{\mathbf{c}}_{3}\right)$ is an involution and multiply with it on the left. Then we may consider the equality of the matrix elements

$$
\left(\hat{\mathbf{c}}_{2}, \mathcal{O}\left(\hat{\mathbf{c}}_{3}\right) \mathcal{R}(\mathbf{c}) \hat{\mathbf{c}}_{1}\right)=\left(\hat{\mathbf{c}}_{2}, \mathcal{R}\left(v \hat{\mathbf{c}}_{2}\right) \mathcal{R}\left(u \hat{\mathbf{c}}_{1}\right) \hat{\mathbf{c}}_{1}\right)
$$

that can be written also as

$$
2 \kappa_{23} \sigma_{31}=\kappa_{12}+\sigma_{21} .
$$

The latter can be used as a test whether $\mathcal{R}\left(\mathbf{c}_{3}\right)$ is actually a half turn. This can be also interpreted as a condition that the product $\mathcal{O}\left(\hat{\mathbf{c}}_{3}\right) \mathcal{R}(\mathbf{c})$ can be decomposed into a pair of rotations with respect to $\hat{\mathbf{c}}_{1}$ and $\hat{\mathbf{c}}_{2}$ in that order.

As for the composition of vector parameters in the divergent case, it is a simple matter of asymptotic behavior. For example in the formula

$$
\left\langle\mathbf{c}_{3}, \mathbf{c}\right\rangle=\frac{w \hat{\mathbf{c}}_{3}+\tau \hat{\mathbf{n}}+w \tau \hat{\mathbf{c}}_{3} \times \hat{\mathbf{n}}}{1-w \tau\left(\hat{\mathbf{c}}_{3}, \hat{\mathbf{n}}\right)}
$$

the limit $w \rightarrow \infty$ depends on two things: whether $\tau$ also diverges and whether $\hat{\mathbf{n}} \perp \hat{\mathbf{c}}_{3}$. The latter corresponds to a half turn about the rotated vector $\hat{\mathbf{c}}_{3}$ while the former indicates that the rotation we decompose is a half turn itself. In that case $(\tau \rightarrow \infty)$ the resultant vector of the composition is

$$
\tilde{\mathbf{c}}=\lim _{w, \tau \rightarrow \infty}\left\langle\mathbf{c}_{3}, \mathbf{c}\right\rangle=\frac{\hat{\mathbf{n}} \times \hat{\mathbf{c}}_{3}}{\left(\hat{\mathbf{n}}, \hat{\mathbf{c}}_{3}\right)}, \quad|\tilde{\mathbf{c}}|= \pm \tan \measuredangle\left(\hat{\mathbf{n}}, \hat{\mathbf{c}}_{3}\right)
$$

and therefore the angle of the compound rotation equals twice the angle between the lines (or planes), determined by $\hat{\mathbf{n}}$ and $\hat{\mathbf{c}}_{3}$, which illustrates an old theorem from elementary geometry.

If $\tau$ is finite on the other hand, the composition law gives

$$
\tilde{\mathbf{c}}=\lim _{w \rightarrow \infty}\left\langle\mathbf{c}_{3}, \mathbf{c}\right\rangle=\frac{\hat{\mathbf{n}} \times \hat{\mathbf{c}}_{3}}{\left(\hat{\mathbf{n}}, \hat{\mathbf{c}}_{3}\right)}-\frac{\hat{\mathbf{c}}_{\mathbf{3}}}{\tau\left(\hat{\mathbf{n}}, \hat{\mathbf{c}}_{3}\right)} .
$$

In each case the problem is reduced to decomposing a rotation, determined by its vector parameter $\tilde{\mathbf{c}}$ given above, into two successive rotations about the axes $\hat{\mathbf{c}}_{1}$ and $\hat{\mathbf{c}}_{2}$. The explicit solution for $u$ and $v$ is given directly by (32)

$$
u=\frac{\left(\rho_{2} \rho_{3}-\kappa_{23}\right) \tau+\tilde{\rho}_{1}}{\left(\rho_{3} \tilde{\rho}_{3}-\rho_{1} \tilde{\rho}_{1}\right) \tau+\kappa_{12} \rho_{3}-\kappa_{23} \rho_{1}}, \quad v=\frac{\left(\kappa_{13}-\rho_{1} \rho_{3}\right) \tau+\tilde{\rho}_{2}}{\left(\rho_{2} \tilde{\rho}_{2}+\rho_{3} \tilde{\rho}_{3}\right) \tau+\kappa_{12} \rho_{3}-\kappa_{13} \rho_{2}} .
$$


In the case

$$
\mathcal{R}(\mathbf{c})=\mathcal{R}\left(w \hat{\mathbf{c}}_{3}\right) \mathcal{R}\left(v \hat{\mathbf{c}}_{2}\right) \mathcal{O}\left(\hat{\mathbf{c}}_{1}\right)
$$

we proceed directly by multiplying on the right with $\mathcal{O}\left(\hat{\mathbf{c}}_{1}\right)$ and considering the scalar product

$$
\left(\hat{\mathbf{c}}_{3}, \mathcal{R}(\mathbf{c}) \mathcal{O}\left(\hat{\mathbf{c}}_{1}\right) \hat{\mathbf{c}}_{2}\right)=\left(\hat{\mathbf{c}}_{3}, \mathcal{R}\left(w \hat{\mathbf{c}}_{3}\right) \mathcal{R}\left(v \hat{\mathbf{c}}_{2}\right) \hat{\mathbf{c}}_{2}\right)
$$

to obtain the test condition

$$
2 \kappa_{12} \sigma_{31}=\kappa_{32}+\sigma_{32}
$$

As for the solutions - they are given by the formulas (34)

$$
v=\frac{\left(\kappa_{13}-\rho_{1} \rho_{3}\right) \tau+\tilde{\rho}_{2}}{\left(\rho_{1} \tilde{\rho}_{1}+\rho_{2} \tilde{\rho}_{2}\right) \tau+\kappa_{23} \rho_{1}-\kappa_{13} \rho_{2}}, \quad w=\frac{\left(\kappa_{12}-\rho_{1} \rho_{2}\right) \tau-\tilde{\rho}_{3}}{\left(\rho_{3} \tilde{\rho}_{3}-\rho_{1} \tilde{\rho}_{1}\right) \tau+\kappa_{12} \rho_{3}-\kappa_{23} \rho_{1}} .
$$

Concerning the vector parameters, the results can be easily derived from $\left\langle\mathbf{c}, \mathbf{c}_{1}\right\rangle$, letting $u \rightarrow \infty$. More precisely, we have

$$
\lim _{u \rightarrow \infty}\left\langle\mathbf{c}, \mathbf{c}_{1}\right\rangle=\frac{\hat{\mathbf{c}}_{1} \times \hat{\mathbf{n}}}{\left(\hat{\mathbf{c}}_{1}, \hat{\mathbf{n}}\right)}-\frac{\hat{\mathbf{c}}_{1}}{\tau\left(\hat{\mathbf{c}}_{1}, \hat{\mathbf{n}}\right)}
$$

for the generic case and

$$
\lim _{u, \tau \rightarrow \infty}\left\langle\mathbf{c}, \mathbf{c}_{1}\right\rangle=\frac{\hat{\mathbf{c}}_{1} \times \hat{\mathbf{n}}}{\left(\hat{\mathbf{c}}_{1}, \hat{\mathbf{n}}\right)}, \quad|\tilde{\mathbf{c}}|= \pm \tan \measuredangle\left(\hat{\mathbf{c}}_{1}, \hat{\mathbf{n}}\right)
$$

for the case when $\mathcal{R}(\mathbf{c})$ is also a half turn.

In the particular case $\mathcal{R}(\mathbf{c})=\mathcal{O}\left(\hat{\mathbf{c}}_{3}\right) \mathcal{O}\left(\hat{\mathbf{c}}_{2}\right) \mathcal{R}\left(u \hat{\mathbf{c}}_{1}\right)$ we only need the asymptotic behavior of (32) in the limit $v \rightarrow \infty$ to obtain (40)

$$
\lim _{v, w \rightarrow \infty} u(v, w)=\frac{\tilde{m} \tau-\omega}{\tilde{\rho}_{1} \tau+\kappa_{23}} .
$$

There are two conditions in this case, namely

$$
2\left(\kappa_{13} \sigma_{31}-\kappa_{12}^{2}\right)=\sigma_{11}-1, \quad\left(\hat{\mathbf{c}}_{2} \times \hat{\mathbf{c}}_{3}, \mathcal{R}(\mathbf{c}) \hat{\mathbf{c}}_{1}\right)=\omega .
$$

The first one follows directly from a suitably chosen scalar product for the equality $\mathcal{O}\left(\hat{\mathbf{c}}_{3}\right) \mathcal{R}(\mathbf{c})=\mathcal{O}\left(\hat{\mathbf{c}}_{2}\right) \mathcal{R}\left(u \hat{\mathbf{c}}_{1}\right)$ and the second one - from the property that $\mathcal{R}$ can be decomposed into a pair of rotations about the axes $\hat{\mathbf{c}}_{1}$ and $\hat{\mathbf{c}}_{2} \times \hat{\mathbf{c}}_{3}$ (the latter may be seen from the composition of vector parameters for the product $\left.\mathcal{O}\left(\hat{\mathbf{c}}_{3}\right) \mathcal{O}\left(\hat{\mathbf{c}}_{2}\right)\right)$. In the case when the denominator vanishes, while the nominator is nonzero, we obviously have a composition of three half turns, that will be discussed later on. 
In a similar way we obtain

$$
2\left(\kappa_{13} \sigma_{31}-\kappa_{23}^{2}\right)=\sigma_{33}-1, \quad\left(\hat{\mathbf{c}}_{3}, \mathcal{R}(\mathbf{c}) \hat{\mathbf{c}}_{1} \times \hat{\mathbf{c}}_{2}\right)=\omega
$$

to be the corresponding conditions for the decomposition of the type $\mathcal{R}(\mathbf{c})=$ $\mathcal{R}\left(w \hat{\mathbf{c}}_{3}\right) \mathcal{O}\left(\hat{\mathbf{c}}_{2}\right) \mathcal{O}\left(\hat{\mathbf{c}}_{1}\right)$. If satisfied, the parameters $w$ can be easily obtained from (14) in the limit $v \rightarrow \infty$ in (40) which produces

$$
\lim _{u, v \rightarrow \infty} w(u, v)=\frac{\tilde{m} \tau-\omega}{\tilde{\rho}_{3} \tau+\kappa_{12}} .
$$

If, on the other hand, both (53) and (54) are fulfilled, the decomposition could be a fact or not as this is not yet a sufficient condition in order to have $\mathcal{R}(\mathbf{c})=$ $\mathcal{O}\left(\hat{\mathbf{c}}_{3}\right) \mathcal{R}\left(v \hat{\mathbf{c}}_{2}\right) \mathcal{O}\left(\hat{\mathbf{c}}_{1}\right)$. In this case the value of the scalar parameter $u$ can easily be found from (13) in the limit $u, w \rightarrow \infty$, which is (40)

$$
\lim _{u, w \rightarrow \infty} v(u, w)=\frac{\tilde{m} \tau-\omega}{\kappa_{13}-\tilde{\rho}_{2} \tau} .
$$

The exact condition for such decomposition may easily be found from the equalities $\mathcal{O}\left(\hat{\mathbf{c}}_{3}\right) \mathcal{R}(\mathbf{c})=\mathcal{R}\left(v \hat{\mathbf{c}}_{2}\right) \mathcal{O}\left(\hat{\mathbf{c}}_{1}\right)$ and $\mathcal{R}(\mathbf{c}) \mathcal{O}\left(\hat{\mathbf{c}}_{1}\right)=\mathcal{O}\left(\hat{\mathbf{c}}_{3}\right) \mathcal{R}\left(v \hat{\mathbf{c}}_{2}\right)$, used in suitable scalar products. The result is

$$
\sigma_{22}-1=2\left(\sigma_{21} \kappa_{21}-\kappa_{23}^{2}\right)=2\left(\sigma_{23} \kappa_{23}-\kappa_{21}^{2}\right) .
$$

In the special case of a decomposition of the type $\mathcal{R}(\mathbf{c})=\mathcal{O}\left(\hat{\mathbf{c}}_{3}\right) \mathcal{O}\left(\hat{\mathbf{c}}_{2}\right) \mathcal{O}\left(\hat{\mathbf{c}}_{1}\right)$ and considering the equality of vector parameters $\left\langle\mathbf{c}_{3}, \mathbf{c}\right\rangle=\left\langle\mathbf{c}_{2}, \mathbf{c}_{1}\right\rangle$ one finds that

$$
\kappa_{12}+\tilde{\rho}_{3} \tau=\kappa_{23}+\tilde{\rho}_{1} \tau=\kappa_{13}-\tilde{\rho}_{2} \tau=0
$$

which can be interpreted as a condition for zero denominators in (40).

Another interesting situation is a half turn, appearing in the middle of the decomposition, i.e.,

$$
\mathcal{R}(\mathbf{c})=\mathcal{R}\left(w \hat{\mathbf{c}}_{3}\right) \mathcal{O}\left(\hat{\mathbf{c}}_{2}\right) \mathcal{R}\left(u \hat{\mathbf{c}}_{1}\right)
$$

When used in a suitable scalar product the above equality yields

$$
\sigma_{31}=2 \kappa_{12} \kappa_{23}-\kappa_{13}
$$

which can be utilized as a test whether or not our decomposition really involves a half turn in the middle. Applying the very same technique as before, we come to the equations in (33)

$$
u=\frac{\left(\kappa_{23}-\rho_{2} \rho_{3}\right) \tau-\tilde{\rho}_{1}}{\left(\rho_{1} \tilde{\rho}_{1}+\rho_{2} \tilde{\rho}_{2}\right) \tau+\kappa_{23} \rho_{1}-\kappa_{13} \rho_{2}}, \quad w=\frac{\left(\kappa_{12}-\rho_{1} \rho_{2}\right) \tau-\tilde{\rho}_{3}}{\left(\rho_{2} \tilde{\rho}_{2}+\rho_{3} \tilde{\rho}_{3}\right) \tau+\kappa_{12} \rho_{3}-\kappa_{13} \rho_{2}} .
$$


It remains to discuss what happens if $\mathcal{R}(\mathbf{c})$ is itself a half turn. In each subcase here, the construction we built at the end of Section 3 will suffice.

In order to answer the question properly, let us consider for the concreteness the case $\mathcal{O}(\hat{\mathbf{n}})=\mathcal{O}\left(\hat{\mathbf{c}}_{3}\right) \mathcal{R}\left(v \hat{\mathbf{c}}_{2}\right) \mathcal{R}\left(u \hat{\mathbf{c}}_{1}\right)$. The condition for which we are looking for can be obtained from a simple geometrical argument which relies on the obvious fact that the rotation $\mathcal{O}\left(\hat{\mathbf{c}}_{3}\right) \mathcal{O}(\hat{\mathbf{n}})$ should be decomposable into a pair of rotations about the first and the second axis in that order. The analogue of (53) for this case yields

$$
2 \kappa_{23} \rho_{1} \rho_{3}=\rho_{1} \rho_{2}+\kappa_{13} \kappa_{23} .
$$

The solutions for $u$ and $v$ are identical to the results in (36)

$$
u=\frac{\rho_{2} \rho_{3}-\kappa_{23}}{\rho_{3} \tilde{\rho}_{3}-\rho_{1} \tilde{\rho}_{1}}, \quad v=\frac{\kappa_{13}-\rho_{1} \rho_{3}}{\rho_{2} \tilde{\rho}_{2}+\rho_{3} \tilde{\rho}_{3}}
$$

as we can rely once more on l'Hôpital's rule, this time in the limit $w \rightarrow \infty$.

For the case $\mathcal{O}(\hat{\mathbf{n}})=\mathcal{R}\left(w \hat{\mathbf{c}}_{3}\right) \mathcal{R}\left(v \hat{\mathbf{c}}_{2}\right) \mathcal{O}\left(\hat{\mathbf{c}}_{1}\right)$, following the same construction, we easily obtain the condition

$$
2 \kappa_{12} \rho_{1} \rho_{3}=\rho_{2} \rho_{3}+\kappa_{12} \kappa_{13}
$$

and the solution is the same as in (38)

$$
v=\frac{\kappa_{13}-\rho_{1} \rho_{3}}{\rho_{1} \tilde{\rho}_{1}+\rho_{2} \tilde{\rho}_{2}}, \quad w=\frac{\rho_{1} \rho_{2}-\kappa_{12}}{\rho_{1} \tilde{\rho}_{1}-\rho_{3} \tilde{\rho}_{3}} .
$$

Finally, if both $v$ and $\tau$ are infinite, that is, if a half turn appears in the middle of the decomposition of another half turn, we consider the equation

$$
\left(\hat{\mathbf{c}}_{3}, \mathcal{R}\left(-w \hat{\mathbf{c}}_{3}\right) \mathcal{O}(\hat{\mathbf{n}}) \hat{\mathbf{c}}_{1}\right)=\left(\hat{\mathbf{c}}_{3}, \mathcal{O}\left(\hat{\mathbf{c}}_{2}\right) \mathcal{R}\left(u \hat{\mathbf{c}}_{1}\right) \hat{\mathbf{c}}_{1}\right)
$$

in order to obtain the equality

$$
\rho_{1} \rho_{3}=\kappa_{12} \kappa_{23}
$$

that can be used as a test condition.

The solutions, once more, can be borrowed this time from (37)

$$
u=\frac{\kappa_{23}-\rho_{2} \rho_{3}}{\rho_{1} \tilde{\rho}_{1}+\rho_{2} \tilde{\rho}_{2}}, \quad w=\frac{\kappa_{12}-\rho_{1} \rho_{2}}{\rho_{2} \tilde{\rho}_{2}+\rho_{3} \tilde{\rho}_{3}} .
$$

In some circumstances, say $\kappa_{12}=\kappa_{23}=0$ and $\boldsymbol{\rho}=0$, which may occur in the classical Euler case of coincident first and third axes, the respective equations 
from (37) becomes useless and the only relation for the two variables (obtained from (13) in the limit $v, \tau \rightarrow \infty$ ) turns out to be

$$
\tilde{\rho}_{1} u w=\tilde{\rho}_{3}
$$

So, in such cases the decomposition depends on an arbitrary scalar parameter and therefore we may, with clear conscious, call such solutions "degenerate". This interesting situation deserves special treatment and will be illustrated by an example further on.

For the cases of two half turns in the decomposition, we use the conditions (55) and (56), written for the symmetric case and double limit in the equations (39), leading to (40) and (41). In the specific case $\mathcal{O}(\hat{\mathbf{n}})=\mathcal{O}\left(\hat{\mathbf{c}}_{3}\right) \mathcal{R}\left(v \hat{\mathbf{c}}_{2}\right) \mathcal{O}\left(\hat{\mathbf{c}}_{1}\right)$, one may use the equalities $\mathcal{O}\left(\hat{\mathbf{c}}_{3}\right) \mathcal{O}(\hat{\mathbf{n}})=\mathcal{R}\left(v \hat{\mathbf{c}}_{2}\right) \mathcal{O}\left(\hat{\mathbf{c}}_{1}\right)$ and $\mathcal{O}(\hat{\mathbf{n}}) \mathcal{O}\left(\hat{\mathbf{c}}_{1}\right)=\mathcal{O}\left(\hat{\mathbf{c}}_{3}\right) \mathcal{R}\left(v \hat{\mathbf{c}}_{2}\right)$ in suitably chosen scalar products to obtain

$$
\tilde{\rho}_{2} \kappa_{23}+\tilde{\rho}_{3}=0, \quad \tilde{\rho}_{2} \kappa_{21}+\tilde{\rho}_{1}=0 .
$$

The explicit solution given by (41) is

$$
u=\frac{\tilde{m}}{\tilde{\rho}_{1}}, \quad v=-\frac{\tilde{m}}{\tilde{\rho}_{2}}, \quad w=\frac{\tilde{m}}{\tilde{\rho}_{3}} .
$$

Similarly, we obtain the remaining two conditions using the same technique as in (55) and the additional equality

$$
\rho_{1} \tilde{\rho}_{1}+\rho_{2} \tilde{\rho}_{2}+\rho_{3} \tilde{\rho}_{3}=\omega .
$$

We have

$$
\rho_{1}^{2}-2 \rho_{1} \rho_{3} \kappa_{13}=1-\kappa_{12}^{2}-\kappa_{13}^{2}, \quad \rho_{2} \tilde{\rho}_{2}+\rho_{3} \tilde{\rho}_{3}=0
$$

for $\mathcal{O}(\hat{\mathbf{n}})=\mathcal{O}\left(\hat{\mathbf{c}}_{3}\right) \mathcal{O}\left(\hat{\mathbf{c}}_{2}\right) \mathcal{R}\left(u \hat{\mathbf{c}}_{1}\right)$ and respectively

$$
\rho_{3}^{2}-2 \rho_{1} \rho_{3} \kappa_{13}=1-\kappa_{13}^{2}-\kappa_{23}^{2}, \quad \rho_{1} \tilde{\rho}_{1}+\rho_{2} \tilde{\rho}_{2}=0
$$

for $\mathcal{O}(\hat{\mathbf{n}})=\mathcal{R}\left(w \hat{\mathbf{c}}_{3}\right) \mathcal{O}\left(\hat{\mathbf{c}}_{2}\right) \mathcal{O}\left(\hat{\mathbf{c}}_{1}\right)$. In particular $\mathcal{O}(\hat{\mathbf{n}})$ can be decomposed into three half turns. Elementary geometrical argument shows that this is only possible if

$$
\tilde{\rho}=0
$$

which is equivalent to the condition that the vectors $\hat{\mathbf{c}}_{k}$ determine a plane and $\hat{\mathbf{n}}$ belongs to it. 


\section{The Identity Transformation}

For the sake of thoroughness we also consider the trivial case $\mathcal{R} \equiv \mathcal{I}$. Since this is the only representative of $\mathrm{SO}(3)$ for which the invariant axis is not unique, it deserves a special treatment. As neither of the objects $\boldsymbol{\rho}, \tilde{\rho}$ and $\boldsymbol{\xi}$ is well defined, we cannot use our main construction (12) here, but we can still take advantage of the vector parameter composition law in three different ways

$$
\mathbf{c}_{1}=\left\langle-\mathbf{c}_{2},-\mathbf{c}_{3}\right\rangle, \quad \mathbf{c}_{2}=\left\langle-\mathbf{c}_{3},-\mathbf{c}_{1}\right\rangle, \quad \mathbf{c}_{3}=\left\langle-\mathbf{c}_{1},-\mathbf{c}_{2}\right\rangle .
$$

The idea is still quite similar to (12) - we multiply the $k^{\text {th }}$ equality in the system with $\hat{\mathbf{c}}_{k}^{\times}$and then consider the scalar products with $\hat{\mathbf{c}}_{2}$ for the first, respectively $\hat{\mathbf{c}}_{3}$ for the second and $\hat{\mathbf{c}}_{1}$ for the third relation

$$
\begin{aligned}
& \left(\kappa_{13}-\kappa_{12} \kappa_{23}\right) v w+\omega w=0 \\
& \left(\kappa_{12}-\kappa_{13} \kappa_{23}\right) u w+\omega u=0 \\
& \left(\kappa_{23}-\kappa_{12} \kappa_{13}\right) u v+\omega v=0 .
\end{aligned}
$$

In this way we guarantee maximal rank, unless $\hat{\mathbf{c}}_{1}$ and $\hat{\mathbf{c}}_{3}$ are parallel, in which case ( or more generally in the case $\omega=0$ ) we have $v=0$, that can also be derived from the particular form of (8) in this setting

$$
\left(\kappa_{31}-\kappa_{12} \kappa_{23}\right) v^{2}+\omega v=0
$$

where the solutions are easily obtained as

$$
v_{-}=0, \quad v_{+}=\frac{\omega}{\kappa_{12} \kappa_{23}-\kappa_{13}} .
$$

Note that two axes decompositions are only possible for the first and the third axis, and only if they are collinear. Then $v_{-}=v_{+}$and we have the trivial $\mathcal{R}_{1}=\mathcal{R}_{3}^{-1}$ so

$$
\hat{\mathbf{e}}_{1} \| \hat{\mathbf{e}}_{3} \Rightarrow \quad v=0
$$

leading to a single relation for the two parameters

$$
u+\kappa_{13} w=0 .
$$

Actually this is another example of a degenerate solution (including half turns as a limiting case) and the only one corresponding to $\omega=0$ (besides the trivial one $u=v=w=0$ ).

Because in the regular case

$$
\omega \neq 0
$$


and as long as no half turns are present in the decomposition, the system (69) produces the solutions $u=v=w=0$, and

$$
u=\frac{\omega}{\kappa_{12} \kappa_{13}-\kappa_{23}}, \quad v=\frac{\omega}{\kappa_{12} \kappa_{23}-\kappa_{13}}, \quad w=\frac{\omega}{\kappa_{13} \kappa_{23}-\kappa_{12}} .
$$

Note that the denominators coincide with the corresponding conditions for the presence of a half turn, which also follows from other considerations, thoroughly discussed in Section 5.2. In particular if

$$
\kappa_{12} \kappa_{23}-\kappa_{13}=0
$$

then we have a decomposition of the type

$$
\mathcal{I}=\mathcal{R}\left(\mathbf{c}_{3}\right) \mathcal{O}\left(\hat{\mathbf{c}}_{2}\right) \mathcal{R}\left(\mathbf{c}_{1}\right) .
$$

The solutions for $u$ and $w$ can still be derived from (75) as long as none of the remaining two parameters diverges. In a similar way we may write

$$
\kappa_{13} \kappa_{23}-\kappa_{12}=0 \Rightarrow \mathcal{I}=\mathcal{O}\left(\hat{\mathbf{c}}_{3}\right) \mathcal{R}\left(\mathbf{c}_{2}\right) \mathcal{R}\left(\mathbf{c}_{1}\right)
$$

or

$$
\kappa_{12} \kappa_{13}-\kappa_{23}=0 \Rightarrow \mathcal{I}=\mathcal{R}\left(\mathbf{c}_{3}\right) \mathcal{R}\left(\mathbf{c}_{2}\right) \mathcal{O}\left(\hat{\mathbf{c}}_{1}\right)
$$

and each time the remaining two (non-divergent) parameters can be found using the formulas in (75).

On the other hand, in the case of two half turns it would be more convenient to derive another system for the scalar parameters, and we do so by considering the dot product of the $k^{t h}$ relation in (68) with $\hat{\mathbf{c}}_{k}$. This allows to write down each of the parameters as a function of the other two

$$
u=\frac{\omega v w-\kappa_{12} v-\kappa_{13} w}{1-\kappa_{23} u v}, \quad v=\frac{\omega u w-\kappa_{12} u-\kappa_{23} w}{1-\kappa_{13} u w}, \quad w=\frac{\omega u v-\kappa_{13} u-\kappa_{23} v}{1-\kappa_{12} u v}
$$

and we can take double limits

$$
\begin{aligned}
& \kappa_{12}=\kappa_{13}=0 \Rightarrow \quad u=\lim _{v, w \rightarrow \infty} u(v, w)=-\frac{\omega}{\kappa_{23}} \\
& \kappa_{12}=\kappa_{23}=0 \Rightarrow \quad v=\lim _{u, w \rightarrow \infty} v(u, w)=-\frac{\omega}{\kappa_{13}} \\
& \kappa_{13}=\kappa_{23}=0 \Rightarrow \quad w=\lim _{u, v \rightarrow \infty} w(u, v)=-\frac{\omega}{\kappa_{12}}
\end{aligned}
$$

provided that the corresponding conditions are fulfilled. Note that the latter can be derived from both geometric and algebraic considerations, as it has been already done in the cases, discussed in Section 5.2. In particular, if

$$
\kappa_{12}=\kappa_{13}=\kappa_{23}=0
$$


we encounter a decomposition into three half turns $\mathcal{I}=\mathcal{O}\left(\hat{\mathbf{c}}_{3}\right) \mathcal{O}\left(\hat{\mathbf{c}}_{2}\right) \mathcal{O}\left(\hat{\mathbf{c}}_{1}\right)$.

With this we exhaust all possibilities for the decomposition of the rotations in $\mathbb{R}^{3}$ into two or three successive rotations about initially given axes. In the next section we illustrate our methods on the well-known examples treated in [20] and [32], as well as some more exotic cases, consistently ignored up to now by other authors. The suggested generalizations and directions for further studies can be found at the end of the paper. An outline of the algorithm is presented as a flowchart in Fig. 1. In Table 2 we have presented all possible decompositions of rotations with respect to three axes, along with the corresponding conditions.

\section{Examples}

A brief formulation of the proposed approach to various decompositions of the rotational matrices is illustrated in Fig. 1. Here we will consider various applications of our scheme (see also [2]).

We will start with the Euler and Bryan decompositions of the symmetric orthogonal matrix

$$
\mathcal{O}(\hat{\mathbf{n}})=\frac{1}{3}\left(\begin{array}{rrr}
-1 & 2 & 2 \\
2 & -1 & 2 \\
2 & 2 & -1
\end{array}\right) \Longrightarrow \hat{\mathbf{n}}^{t}=\frac{1}{\sqrt{3}}(1,1,1)
$$

which plays an essential role in the construction of the minimal atlas for the rotational group $\mathrm{SO}(3)$ [11]. Our concern here will be the decomposition of this half turn rotation into three consecutive rotations about the axes

$$
\hat{\mathbf{c}}_{1}^{t}=\frac{1}{\sqrt{3}}(1,-1,-1), \quad \hat{\mathbf{c}}_{2}^{t}=\frac{1}{\sqrt{3}}(-1,1,-1), \quad \hat{\mathbf{c}}_{3}^{t}=\frac{1}{\sqrt{3}}(-1,-1,1) .
$$

Using the algorithm described above, we obtain the scalar parameters $(u, v, w)$, the angles of rotation (in degrees)

$$
\boldsymbol{\psi}^{t}=\frac{180^{\circ}}{\pi}(2 \arctan u, 2 \arctan v, 2 \arctan w)
$$

and thus the matrix decomposition (7).

For the so chosen matrix (81) we obtain, via the techniques described in Section 2, only one solution $(u, v, w)=\left(-\sqrt{3}, \frac{1}{\sqrt{3}},-\sqrt{3}\right)$ since equation (8) has zero discriminant. For the respective angles we have

$$
\left(\psi_{1}, \psi_{2}, \psi_{3}\right)=\left(-120^{\circ}, 60^{\circ},-120^{\circ}\right)
$$


Table 2. Three axes decompositions. $(*)$ degenerate one parameter families of solutions are possible, in which there is a single nontrivial relation for the two unknowns. (**) degenerate solutions, corresponding to mutually inverse rotations about parallel axes.

\begin{tabular}{|c|c|c|c|c|}
\hline Decomposition & Criteria & $u$ & $v$ & $w$ \\
\hline $\mathcal{R}(\mathbf{c})=\mathcal{R}\left(w \hat{\mathbf{c}}_{3}\right) \mathcal{R}\left(v \hat{\mathbf{c}}_{2}\right) \mathcal{R}\left(u \hat{\mathbf{c}}_{1}\right)$ & (9) & (13) & (16) & (14) \\
\hline $\mathcal{R}(\mathbf{c})=\mathcal{O}\left(\hat{\mathbf{c}}_{3}\right) \mathcal{R}\left(v \hat{\mathbf{c}}_{2}\right) \mathcal{R}\left(u \hat{\mathbf{c}}_{1}\right)$ & (53) & (32) & $(32)$ & $\infty$ \\
\hline $\mathcal{R}(\mathbf{c})=\mathcal{R}\left(w \hat{\mathbf{c}}_{3}\right) \mathcal{O}\left(\hat{\mathbf{c}}_{2}\right) \mathcal{R}\left(u \hat{\mathbf{c}}_{1}\right)$ & (59) & (33) & $\infty$ & (33) \\
\hline $\mathcal{R}(\mathbf{c})=\mathcal{R}\left(w \hat{\mathbf{c}}_{3}\right) \mathcal{R}\left(v \hat{\mathbf{c}}_{2}\right) \mathcal{O}\left(\hat{\mathbf{c}}_{1}\right)$ & (54) & $\infty$ & (34) & (34) \\
\hline $\mathcal{R}(\mathbf{c})=\mathcal{O}\left(\hat{\mathbf{c}}_{3}\right) \mathcal{O}\left(\hat{\mathbf{c}}_{2}\right) \mathcal{R}\left(u \hat{\mathbf{c}}_{1}\right)$ & $(55)$ & (40) & $\infty$ & $\infty$ \\
\hline $\mathcal{R}(\mathbf{c})=\mathcal{R}\left(w \hat{\mathbf{c}}_{3}\right) \mathcal{O}\left(\hat{\mathbf{c}}_{2}\right) \mathcal{O}\left(\hat{\mathbf{c}}_{1}\right)$ & $(56)$ & $\infty$ & $\infty$ & $(40)$ \\
\hline $\mathcal{R}(\mathbf{c})=\mathcal{O}\left(\hat{\mathbf{c}}_{3}\right) \mathcal{R}\left(v \hat{\mathbf{c}}_{2}\right) \mathcal{O}\left(\hat{\mathbf{c}}_{1}\right)$ & $(54),(57)$ & $\infty$ & $(40)$ & $\infty$ \\
\hline $\mathcal{R}(\mathbf{c})=\mathcal{O}\left(\hat{\mathbf{c}}_{3}\right) \mathcal{O}\left(\hat{\mathbf{c}}_{2}\right) \mathcal{O}\left(\hat{\mathbf{c}}_{1}\right)$ & $(55),(58)$ & $\infty$ & $\infty$ & $\infty$ \\
\hline $\mathcal{O}(\hat{\mathbf{n}})=\mathcal{R}\left(w \hat{\mathbf{c}}_{3}\right) \mathcal{R}\left(v \hat{\mathbf{c}}_{2}\right) \mathcal{R}\left(u \hat{\mathbf{c}}_{1}\right)$ & (9) & (23) & (20) & (23) \\
\hline $\mathcal{O}(\hat{\mathbf{n}})=\mathcal{O}\left(\hat{\mathbf{c}}_{3}\right) \mathcal{R}\left(v \hat{\mathbf{c}}_{2}\right) \mathcal{R}\left(u \hat{\mathbf{c}}_{1}\right)$ & $(60)$ & (36) & (36) & $\infty$ \\
\hline $\mathcal{O}(\hat{\mathbf{n}})=\mathcal{R}\left(w \hat{\mathbf{c}}_{3}\right) \mathcal{O}\left(\hat{\mathbf{c}}_{2}\right) \mathcal{R}\left(u \hat{\mathbf{c}}_{1}\right)$ & $(62)$ & $(37),(63)(*)$ & $\infty$ & $(37),(63)(*)$ \\
\hline $\mathcal{O}(\hat{\mathbf{n}})=\mathcal{R}\left(w \hat{\mathbf{c}}_{3}\right) \mathcal{R}\left(v \hat{\mathbf{c}}_{2}\right) \mathcal{O}\left(\hat{\mathbf{c}}_{1}\right)$ & $(61)$ & $\infty$ & $(38)$ & $(38)$ \\
\hline $\mathcal{O}(\hat{\mathbf{n}})=\mathcal{O}\left(\hat{\mathbf{c}}_{3}\right) \mathcal{O}\left(\hat{\mathbf{c}}_{2}\right) \mathcal{R}\left(u \hat{\mathbf{c}}_{1}\right)$ & $(65)$ & $(41)$ & $\infty$ & $\infty$ \\
\hline $\mathcal{O}(\hat{\mathbf{n}})=\mathcal{R}\left(w \hat{\mathbf{c}}_{3}\right) \mathcal{O}\left(\hat{\mathbf{c}}_{2}\right) \mathcal{O}\left(\hat{\mathbf{c}}_{1}\right)$ & (66) & $\infty$ & $\infty$ & (41) \\
\hline $\mathcal{O}(\hat{\mathbf{n}})=\mathcal{O}\left(\hat{\mathbf{c}}_{3}\right) \mathcal{R}\left(v \hat{\mathbf{c}}_{2}\right) \mathcal{O}\left(\hat{\mathbf{c}}_{1}\right)$ & $(64)$ & $\infty$ & $(41)$ & $\infty$ \\
\hline $\mathcal{O}(\hat{\mathbf{n}})=\mathcal{O}\left(\hat{\mathbf{c}}_{3}\right) \mathcal{O}\left(\hat{\mathbf{c}}_{2}\right) \mathcal{O}\left(\hat{\mathbf{c}}_{1}\right)$ & $(67)$ & $\infty$ & $\infty$ & $\infty$ \\
\hline$\overline{\mathcal{I}}=\mathcal{R}\left(w \hat{\mathbf{c}}_{3}\right) \mathcal{R}\left(v \hat{\mathbf{c}}_{2}\right) \mathcal{R}\left(u \hat{\mathbf{c}}_{1}\right)$ & $(74)$ & $(75)$ & $(75)$ & $(75)$ \\
\hline $\mathcal{I}=\mathcal{O}\left(\hat{\mathbf{c}}_{3}\right) \mathcal{R}\left(v \hat{\mathbf{c}}_{2}\right) \mathcal{R}\left(u \hat{\mathbf{c}}_{1}\right)$ & $(74),(77)$ & $(75)$ & $(75)$ & $\infty$ \\
\hline $\mathcal{I}=\mathcal{R}\left(w \hat{\mathbf{c}}_{3}\right) \mathcal{O}\left(\hat{\mathbf{c}}_{2}\right) \mathcal{R}\left(u \hat{\mathbf{c}}_{1}\right)$ & $(74),(77)$ & (75) & $\infty$ & (75) \\
\hline $\mathcal{I}=\mathcal{R}\left(w \hat{\mathbf{c}}_{3}\right) \mathcal{R}\left(v \hat{\mathbf{c}}_{2}\right) \mathcal{O}\left(\hat{\mathbf{c}}_{1}\right)$ & $(74),(77)$ & $\infty$ & $(75)$ & $(75)$ \\
\hline $\mathcal{I}=\mathcal{O}\left(\hat{\mathbf{c}}_{3}\right) \mathcal{O}\left(\hat{\mathbf{c}}_{2}\right) \mathcal{R}\left(u \hat{\mathbf{c}}_{1}\right)$ & $(74),(79)$ & (79) & $\infty$ & $\infty$ \\
\hline $\mathcal{I}=\mathcal{R}\left(w \hat{\mathbf{c}}_{3}\right) \mathcal{O}\left(\hat{\mathbf{c}}_{2}\right) \mathcal{O}\left(\hat{\mathbf{c}}_{1}\right)$ & $(74),(79)$ & $\infty$ & $\infty$ & $(79)$ \\
\hline $\mathcal{I}=\mathcal{O}\left(\hat{\mathbf{c}}_{3}\right) \mathcal{R}\left(v \hat{\mathbf{c}}_{2}\right) \mathcal{O}\left(\hat{\mathbf{c}}_{1}\right)$ & $(74),(79)$ & $\infty$ & $(79)$ & $\infty$ \\
\hline $\mathcal{I}=\mathcal{O}\left(\hat{\mathbf{c}}_{3}\right) \mathcal{O}\left(\hat{\mathbf{c}}_{2}\right) \mathcal{O}\left(\hat{\mathbf{c}}_{1}\right)$ & $(74),(80)$ & $\infty$ & $\infty$ & $\infty$ \\
\hline $\mathcal{I}=\mathcal{R}\left(w \hat{\mathbf{c}}_{3}\right) \mathcal{R}\left(u \hat{\mathbf{c}}_{1}\right)$ & $(72)$ & $(73)(* *)$ & 0 & $(73)(* *)$ \\
\hline $\mathcal{I}=\mathcal{O}\left(\hat{\mathbf{c}}_{3}\right) \mathcal{O}\left(\hat{\mathbf{c}}_{1}\right)$ & $(72)$ & $\infty$ & 0 & $\infty$ \\
\hline
\end{tabular}

and the corresponding rotations about the fixed axes are

$$
\mathcal{R}_{1}=\left(\begin{array}{rrr}
0 & -1 & 0 \\
0 & 0 & 1 \\
-1 & 0 & 0
\end{array}\right), \quad \mathcal{R}_{2}=\frac{1}{3}\left(\begin{array}{rrr}
2 & 1 & 2 \\
-2 & 2 & 1 \\
-1 & -2 & 2
\end{array}\right), \quad \mathcal{R}_{3}=\left(\begin{array}{rrr}
0 & 1 & 0 \\
0 & 0 & -1 \\
-1 & 0 & 0
\end{array}\right)
$$


However, there is one more solution, which corresponds to a half turn, appearing in the middle of the decomposition as it is easy to see that the condition (62) is fulfilled, and since $\kappa_{12} \neq 0$, we can resolve for the unknown $u$ in order to $w$ and obtain $(u, v, w)=\left(\frac{\sqrt{3}}{2}, \infty, \frac{\sqrt{3}}{2}\right)$, or $\psi^{t}=\left(81.785^{\circ}, 180^{\circ}, 81.785^{\circ}\right)$, leading to $\mathcal{R}_{1}=\frac{1}{7}\left(\begin{array}{rrr}3 & -2 & -6 \\ 6 & 3 & 2 \\ 2 & -6 & 3\end{array}\right), \quad \mathcal{R}_{2}=\frac{1}{3}\left(\begin{array}{rrr}-1 & -2 & 2 \\ -2 & -1 & -2 \\ 2 & -2 & -1\end{array}\right), \quad \mathcal{R}_{3}=\frac{1}{7}\left(\begin{array}{rrr}3 & 2 & -6 \\ -6 & 3 & -2 \\ 2 & 6 & 3\end{array}\right)$ which covers the Bryan case. It is necessary to mention also that all even permutations of the axes give the same angles of rotation, while for the odd ones we only need to invert the sign.

As for the Euler case, we use the above set of axes with the modification $\hat{\mathbf{c}}_{3} \equiv \hat{\mathbf{c}}_{1}$. Here we find only one solution $(u, v, w)=(-\sqrt{3}, \infty, \sqrt{3})$, that can be written with the help of the trigonometric formulas as $\boldsymbol{\psi}^{t}=\left(-120^{\circ}, 180^{\circ}, 120^{\circ}\right)$ and which matrix forms are

$$
\mathcal{R}_{1}=\left(\begin{array}{rrr}
0 & -1 & 0 \\
0 & 0 & 1 \\
-1 & 0 & 0
\end{array}\right), \quad \mathcal{R}_{2}=\frac{1}{3}\left(\begin{array}{rrr}
-1 & -2 & 2 \\
-2 & -1 & -2 \\
2 & -2 & -1
\end{array}\right), \quad \mathcal{R}_{3}=\left(\begin{array}{rrr}
0 & 0 & -1 \\
-1 & 0 & 0 \\
0 & 1 & 0
\end{array}\right) .
$$

Next we consider a rotation about the diagonal of the first octant (so $\hat{\mathbf{n}}$ is as in the previous example) by an angle $\varphi=\frac{2 \pi}{3}$ and look for its decomposition into two successive rotations by angles $\psi_{1}=\psi_{2}=\frac{\pi}{2}$ about $O Y, O X$ and $O Z$ axes in that order. There is a pair of twofold solutions since we have two equality relations - both $\kappa_{21}=\sigma_{21}=0$ and $\kappa_{32}=\sigma_{32}=0$. For the first one we have $(u, v, w)=(1,1,0)$ and thus $\boldsymbol{\psi}=\left(90^{\circ}, 90^{\circ}, 0^{\circ}\right)^{t}$ which gives the decomposition in explicit form as

$$
\mathcal{R}=\left(\begin{array}{lll}
0 & 0 & 1 \\
1 & 0 & 0 \\
0 & 1 & 0
\end{array}\right)=\left(\begin{array}{rrr}
1 & 0 & 0 \\
0 & 0 & -1 \\
0 & 1 & 0
\end{array}\right)\left(\begin{array}{rrr}
0 & 0 & 1 \\
0 & 1 & 0 \\
-1 & 0 & 0
\end{array}\right)=\mathcal{R}_{2} \mathcal{R}_{1}
$$

while for the second solution $(u, v, w)=(0,1,1), \boldsymbol{\psi}^{t}=\left(0^{\circ}, 90^{\circ}, 90^{\circ}\right)$ and the matrix decomposition can be written as

$$
\mathcal{R}=\left(\begin{array}{lll}
0 & 0 & 1 \\
1 & 0 & 0 \\
0 & 1 & 0
\end{array}\right)=\left(\begin{array}{rrr}
0 & -1 & 0 \\
1 & 0 & 0 \\
0 & 0 & 1
\end{array}\right)\left(\begin{array}{rrr}
1 & 0 & 0 \\
0 & 0 & -1 \\
0 & 1 & 0
\end{array}\right)=\mathcal{R}_{3} \mathcal{R}_{2} .
$$

Apart from these two solutions, we have two more involving half turns. For example, if we test whether decomposition in the form (52) is possible, we find that it is, 
since the condition (53) is fulfilled and no more half turns are allowed because the orthogonality conditions do not hold, so finally we obtain $(u, v, w)=(-1,1, \infty)$, $\psi^{t}=\left(-90^{\circ}, 90^{\circ}, 180^{\circ}\right)$ and the matrices in the decomposition turns out to be

$$
\mathcal{R}_{1}=\left(\begin{array}{rrr}
0 & 0 & -1 \\
0 & 1 & 0 \\
1 & 0 & 0
\end{array}\right), \quad \mathcal{R}_{2}=\left(\begin{array}{rrr}
1 & 0 & 0 \\
0 & 0 & -1 \\
0 & 1 & 0
\end{array}\right), \quad \mathcal{R}_{3}=\left(\begin{array}{rrr}
-1 & 0 & 0 \\
0 & -1 & 0 \\
0 & 0 & 1
\end{array}\right) .
$$

It turns out that a half turn may appear in the decomposition on the right as well. Applying the same procedure (described in Section 5.2), we obtain $(u, v, w)=$ $(\infty, 1,-1), \boldsymbol{\psi}^{t}=\left(180^{\circ}, 90^{\circ},-90^{\circ}\right)$ and thus

$$
\mathcal{R}_{1}=\left(\begin{array}{rrr}
-1 & 0 & 0 \\
0 & 1 & 0 \\
0 & 0 & -1
\end{array}\right), \quad \mathcal{R}_{2}=\left(\begin{array}{rrr}
1 & 0 & 0 \\
0 & 0 & -1 \\
0 & 1 & 0
\end{array}\right), \quad \mathcal{R}_{3}=\left(\begin{array}{rrr}
0 & 1 & 0 \\
-1 & 0 & 0 \\
0 & 0 & 1
\end{array}\right)
$$

The presence of a half turn in the middle, however, is not an option in this case as (59) is not satisfied, so this gives all possible solutions.

One more exotic example considers the Euler decomposition about the axes $O Y$, $O X$ and $O Y$ in this order of the half turn $\mathcal{O}(\hat{\mathbf{n}})$, where $\hat{\mathbf{n}}^{t}=(0,0,1)$. Using (62) one may easily check that a half turn is involved as a middle factor. The interesting part is that the solutions in this case are infinitely many, depending on one scalar parameter, since we use only one relation (62) for the two parameters. More precisely, for any real number $u \neq 0$, setting $w=-\frac{1}{u}$, we obtain a solution. This means that for any $\phi \neq k \pi, \boldsymbol{\psi}^{t}=(\phi, \pi, \phi-\pi)$ is a solution and the corresponding matrix decomposition is

$$
\mathcal{R}=\left(\begin{array}{rrr}
-1 & 0 & 0 \\
0 & -1 & 0 \\
0 & 0 & 1
\end{array}\right)=\left(\begin{array}{ccr}
-\cos \phi & 0 & \sin \phi \\
0 & 1 & 0 \\
-\sin \phi & 0 & -\cos \phi
\end{array}\right)\left(\begin{array}{rrr}
1 & 0 & 0 \\
0 & -1 & 0 \\
0 & 0 & -1
\end{array}\right)\left(\begin{array}{ccc}
\cos \phi & 0 & -\sin \phi \\
0 & 1 & 0 \\
\sin \phi & 0 & \cos \phi
\end{array}\right)
$$

In particular letting $u \rightarrow \infty$ makes $w$ vanish and vice versa. In this way we obtain the twofold solutions $(u, v, w)=(\infty, \infty, 0)$ and $(u, v, w)=(0, \infty, \infty)$ with the corresponding angular parameters $\boldsymbol{\psi}^{t}=(\pi, \pi, 0)$ and $\boldsymbol{\psi}^{t}=(0, \pi, \pi)$ respectively, as a limiting case of the one parameter family, described above. The corresponding matrix decompositions are

$$
\mathcal{R}=\left(\begin{array}{rrr}
-1 & 0 & 0 \\
0 & -1 & 0 \\
0 & 0 & 1
\end{array}\right)=\left(\begin{array}{rrr}
1 & 0 & 0 \\
0 & -1 & 0 \\
0 & 0 & -1
\end{array}\right)\left(\begin{array}{rrr}
-1 & 0 & 0 \\
0 & 1 & 0 \\
0 & 0 & -1
\end{array}\right)=\mathcal{R}_{2} \mathcal{R}_{1}
$$


for the first case and

$$
\mathcal{R}=\left(\begin{array}{rrr}
-1 & 0 & 0 \\
0 & -1 & 0 \\
0 & 0 & 1
\end{array}\right)=\left(\begin{array}{rrr}
-1 & 0 & 0 \\
0 & 1 & 0 \\
0 & 0 & -1
\end{array}\right)\left(\begin{array}{rrr}
1 & 0 & 0 \\
0 & -1 & 0 \\
0 & 0 & -1
\end{array}\right)=\mathcal{R}_{3} \mathcal{R}_{2}
$$

for the second one.

Our last example is from [32] and [20]. Following these papers, we consider a rotation by an angle $\varphi=60^{\circ}$ about the axis, given by the vector $\hat{\mathbf{n}}$ with coordinates on the unit sphere $(\theta, \phi)=\left(50^{\circ}, 25^{\circ}\right)$. The convention we are using here for the azimuthal angle $\theta \in\left[-\frac{\pi}{2}, \frac{\pi}{2}\right]$, is adopted from [32], which assumes that it is zero at the equator and $90^{\circ}$ at the North Pole. The three axes of rotation are chosen as follows: the first one is $O X$, the second one is $O Y$, rotated by $60^{\circ}$ clockwise in the $X O Y$ plane, and the third one - again $O X$. With five digits precision our scheme produces the following results for the angular variables

$$
\begin{aligned}
& \left(\psi_{1}^{+}, \psi_{2}^{+}, \psi_{3}^{+}\right)=\left(178.50326^{\circ},-108.73792^{\circ},-40.54766^{\circ}\right) \\
& \left(\psi_{1}^{-}, \psi_{2}^{-}, \psi_{3}^{-}\right)=\left(-102.27231^{\circ}, 108.73792^{\circ}, 38.67676^{\circ}\right)
\end{aligned}
$$

which are in complete accordance with the results presented in [32]. If we choose for the third rotation the axis given by a vector with coordinates $(\theta, \phi)=\left(80^{\circ}, 45^{\circ}\right)$ on the unit sphere and keep everything else as before, the results become

$$
\begin{aligned}
& \left(\psi_{1}^{+}, \psi_{2}^{+}, \psi_{3}^{+}\right)=\left(-139.78921^{\circ}, 179.27102^{\circ},-12.20974^{\circ}\right) \\
& \left(\psi_{1}^{-}, \psi_{2}^{-}, \psi_{3}^{-}\right)=\left(33.72840^{\circ},-4.496982^{\circ}, 48.63548^{\circ}\right)
\end{aligned}
$$

which again agree completely with those presented in [32] and [20]. For the scalar parameters we have respectively

$$
\begin{aligned}
& \left(u_{+}, v_{+}, w_{+}\right)=(-2.73183,157.19197,-0.10695) \\
& \left(u_{-}, v_{-}, w_{-}\right)=(0.30314,-0.03926,0.45189)
\end{aligned}
$$

for the first rotation and

$$
\begin{aligned}
& \left(u_{+}, v_{+}, w_{+}\right)=(76.55670,-1.39519,-0.36939) \\
& \left(u_{-}, v_{-}, w_{-}\right)=(-1.24092,1.39519,0.350947)
\end{aligned}
$$

for the second one. These same numbers are obtained in [20] as well, but in order to comply with the notations used there, they are given here in the reverse order. 


\section{Concluding Remarks}

In this article we cover all possibilities for the decomposition of a rotational matrix into two or three rotations about fixed, initially given axes, following the order they are given in.

Unlike other articles on the problem, the present one gives explicit solutions also in the case when some of the scalar parameters in the decomposition become infinite or vanish.

The procedure of obtaining these solutions is linear, except in some cases for the middle parameter, for which one may need to solve a simple quadratic equation. The method is fast and efficient, despite of the various specific cases, which should be treated separately.

Following the algorithm described above and using any modern programming language (we used MAPLE in our tests), one may easily write a routine, that generates all possible solutions, and eventually selects the most efficient one.

Since the efficiency is a priority as far as applications are concerned, one may also consider permutations of the given rotational axes, as an addition to the receipt suggested here, in order to generate more solutions to choose from. However, this is rather trivial upgrade, so we leave it without further discussion.

Another question to ask in this context is if we can generalize our results to $\mathrm{O}(3)$ and the answer is quite easy - the orthogonal matrices with determinant equal to -1 can be mapped to $\mathrm{SO}(3)$ by a simple multiplication with $-\mathcal{I}$. Therefore, all our relations remain valid in principle for this more general case, with one slight inconvenience that we end up with four times more solutions due to the fact that the factor $-\mathcal{I}$ can be assigned to any of the three rotations, or to all of them simultaneously. So, we get four times more solutions in this case.

There are many more possible extensions or generalizations. For example relying on moving frames, as practice very often dictates [13] or lifting to the universal covering group $\mathrm{SU}(2)$ via the use of quaternions $[3,4]$. Another almost straightforward generalization is the expanding of the above results to the dual groups like $\mathrm{SO}(2,1)$ and $\mathrm{SU}(1,1)[9]$.

We leave these and many other unanswered questions and problems for further investigations.

\section{Acknowledgements}

All authors are grateful to Dr Mariana Hadzhilazova for her help with the figure and overall typesetting of the manuscript. 


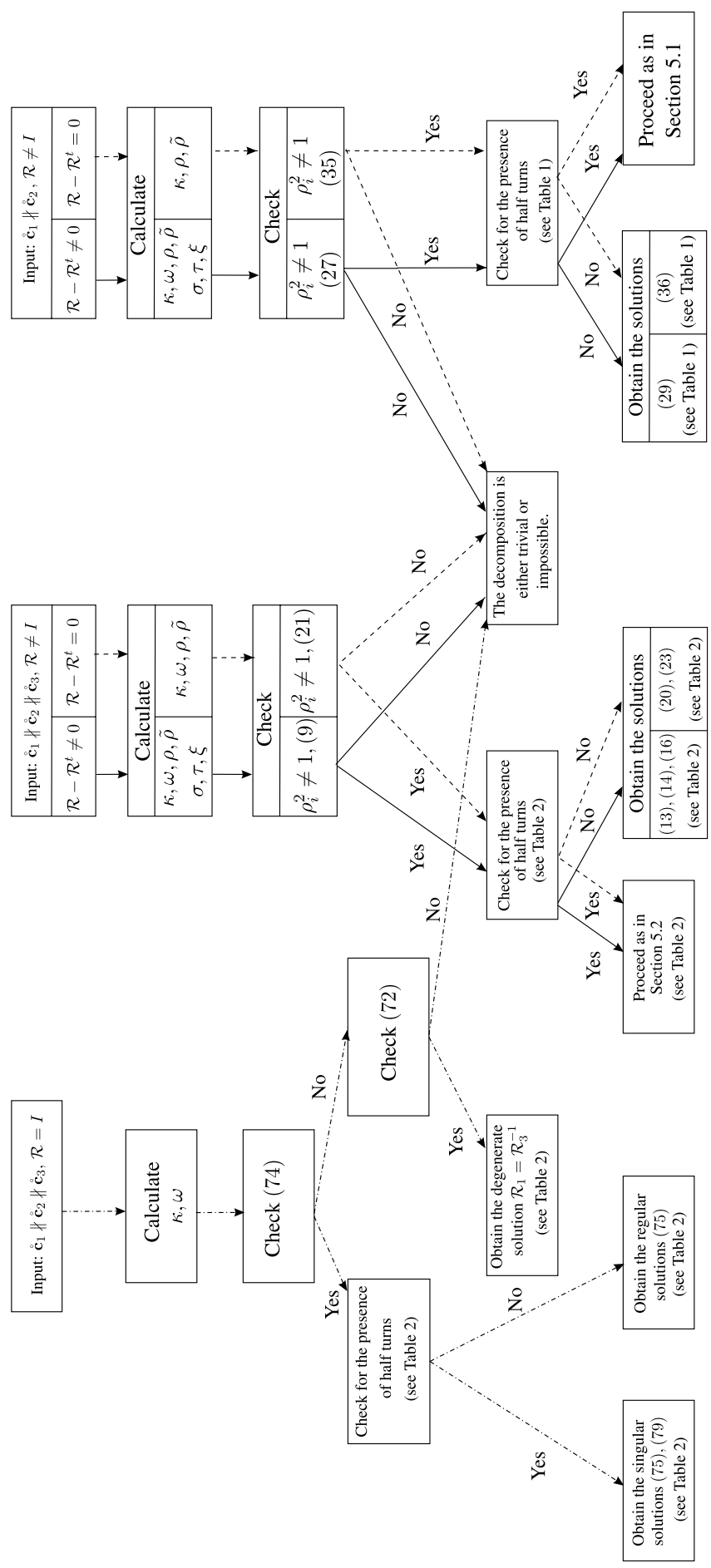

Figure 1. Flow sheet for decomposition of the rotational matrices. 


\section{References}

[1] Borri M., Trainelli L. and Bottaso C., On Representations and Parameterizations of Motion, Multibody System Dynamics 4 (2000) 129-193.

[2] Brezov D., Mladenova C. and Mladenov I., Vector Decomposition of Half Turns, In: Proceedings of the IVII-nd Spring Conference of the Union of the Bulgarian Mathematicians (to appear), Sofia 2013, pp xxx-zzz.

[3] Chobanov G. and Chobanov I., Quaternions and Rotations I. Finite Dispalcements and Finite Rotations, Annuaire de l'Universitè de Sofia - Mathématiques 79 (1985) 15-57.

[4] Chobanov G. and Chobanov I., Quaternions and Rotations II. Quaternions and Finite Rotations, Annuaire de l'Universitè de Sofia - Mathématiques 79 (1985) 59-102.

[5] Davenport P., Rotations About Nonorthogonal Axes, AIAA Journal 11 (1973) 853-857.

[6] Diamond R., On the Factorization of Rotations with Examples in Diffractometry, Proc. R. Soc. Lond. A 428 (1990) 451-472.

[7] Fedorov F., The Lorentz Group (in Russian), Science, Moskow 1979.

[8] Fraiture L., A History of the Description of the Three-Dimensional Finite Rotation, J. Astronautical Sciences 57 (2009) 207-232.

[9] Gilmore R., Relations Among Low-dimensional Simple Lie Groups, J. Geom. Symmetry Phys. 28 (2012) 1-45.

[10] Goldstein H., Classical Mechanics, Addison-Wesley, Reading 1980.

[11] Grafarend E. and Kühnel W., A Minimal Atlas for the Rotation Group $S O(3)$, Int. J. Geomath. 2 (2011) 113-122.

[12] Jazar R., Advanced Dynamics: Rigid Body, Multibody, and Aerospace Applications, Wiley, Hoboken 2011.

[13] Kuipers J., Quaternions and Rotation Sequences, Geometry, Integrability and Quantization 1 (2000) 127-143.

[14] Lenz R., Group Theoretical Methods in Image Processing, Springer, Berlin 1990.

[15] Mladenov I., An Extension of the Saxon-Hutner Theorem in the Relativistic Domain, Phys. Lett. A 137 (1989) 313-318.

[16] Mladenov I., On the Saxon-Hutner Theorem, C. R. Bulg. Acad. Sci. 38 (1985) 993-996.

[17] Mladenov I. and Vassileva J., A Note on Hibridization, Commun. Math. Chem. 11 (1981) 69-73. 
[18] Mladenova C., Group Theory in the Problems of Modeling and Control of Multi-Body Systems, J. Geom. Symmetry Phys. 8 (2006) 17-121.

[19] Mladenova C., An Approach to Description of a Rigid Body Motion, C. R. Acad. Sci. Bulg. 38 (1985) 1657-1660.

[20] Mladenova C. and Mladenov I., Vector Decomposition of Finite Rotations, Rep. Math. Phys. 68 (2011) 107-117.

[21] Mladenova C. and Mladenov I., Spacecraft Dynamics Under the Influence of Gravity Tourques, JTAM 38 (2008) 3-22.

[22] Müller A., Group Theoretical Approaches to Vector Parameterization of Rotations, J. Geom. Symmetry Phys. 19 (2010) 43-72.

[23] Palais B., Palais R. and Rodi S., A Disorienting Look at Euler's Theorem on the Axis of a Rotation, Am. Math. Monthly 116 (2009) 892-909.

[24] Perelyaev S., About the Global Parameterizations of the Group of ThreeDimensional Rotations (in Russian), Rigid Body Mechanics 3 (2006) 30-44.

[25] Pina E., Rotations with Rodrigues' Vector, Eur. J. Phys. 32 (2011)1171-1178.

[26] Piovan G. and Bullo F., On Coordinate-Free Rotation Decomposition Euler Angles About Arbitrary Axes, IEEE Transactions on Robotics 28 (2012) 728733.

[27] Red'kov V. and Tokarevskaya N., Factorizations for 3-rotations and Polarization of the Light in Mueller-Stokes and Jones Formalisms, http://arxiv.org/abs/0808.0852v1.

[28] Rodrigues O., Des lois géométriques qui regissent les déplacéments d'un systéme solide dans l'espace, et de la variation des coordonnées provenant de ces déplacéments considérés indépendamment des causes qui peuvent les produire, J. Math. Pures Appl. 5 (1840) 380-440.

[29] Segercrantz J., New Parameters for Rotations of Solid Bodies, Commentationes Physico-Mathematicae, Societas Scientiarum Fennica 33 (1966) 1-8.

[30] Siminovitch D., Rotations in NMR: Part I. Euler-Rodrigues Parameters and Quaternions, Concepts Magn. Reson. 9 (1997) 149-171.

[31] Stuelpnagel J., On the Parameterization of the Three-Dimensional Rotation Group, SIAM Review 6 (1964) 422-430.

[32] Wohlhart K., Decomposition of a Finite Rotation into Three Consecutive Rotations About Given Axes, In: Proc. VI-th Int Conf. on Theory of Machines and Mechanisms, Czechoslovakia, Liberec 1992, pp 325-332. 
Danail S. Brezov

University of Architecture

Civil Engineering and Geodesy

1 Hristo Smirnenski Blvd.

1046 Sofia, Bulgaria

E-mail address: danail.brezov@gmail.com

Clementina D. Mladenova

Institute of Mechanics

Bulgarian Academy of Sciences

Acad. G. Bonchev Str., Bl. 4

1113 Sofia, Bulgaria

E-mail address: cleme imbm.bas.bg

Ivaïlo M. Mladenov

Institute of Biophysics

Bulgarian Academy of Sciences

Acad. G. Bonchev Str., Bl. 21

1113 Sofia, Bulgaria

E-mail address: mladenov@bio21.bas.bg 